\title{
FIMP dark matter candidate(s) in a $B-L$ model with inverse seesaw mechanism
}

\author{
Waleed Abdallah, ${ }^{a, b}$ Sandhya Choubey ${ }^{a, c}$ and Sarif Khan ${ }^{a, d}$ \\ ${ }^{a}$ Harish-Chandra Research Institute, HBNI, \\ Chhatnag Road, Jhunsi, Allahabad 211019, India \\ ${ }^{b}$ Department of Mathematics, Faculty of Science, Cairo University, \\ Giza 12613, Egypt \\ ${ }^{c}$ Department of Physics, School of Engineering Sciences, \\ KTH Royal Institute of Technology, AlbaNova University Center, \\ 10691 Stockholm, Sweden \\ ${ }^{d}$ Institut für Theoretische Physik, Georg-August-Universität Göttingen, \\ Friedrich-Hund-Platz 1, Göttingen, D-37077 Germany \\ E-mail: waleedabdallah@hri.res.in, sandhya@hri.res.in, \\ sarifkhan@hri.res.in
}

ABSTRACT: The non-thermal dark matter (DM) production via the so-called freeze-in mechanism provides a simple alternative to the standard thermal WIMP scenario. In this work, we consider a popular $\mathrm{U}(1)_{B-L}$ extension of the standard model (SM) in the context of inverse seesaw mechanism which has at least one (fermionic) FIMP DM candidate. Due to the added $\mathbb{Z}_{2}$ symmetry, a SM gauge singlet fermion, with mass of order keV, is stable and can be a warm DM candidate. Also, the same $\mathbb{Z}_{2}$ symmetry helps the lightest righthanded neutrino, with mass of order $\mathrm{GeV}$, to be a stable or long-lived particle by making a corresponding Yukawa coupling very small. This provides a possibility of a two component DM scenario as well. Firstly, in the absence of a GeV DM component (i.e., without tuning its corresponding Yukawa coupling to be very small), we consider only a keV DM as a single component DM, which is produced by the freeze-in mechanism via the decay of the extra $Z^{\prime}$ gauge boson associated to $\mathrm{U}(1)_{B-L}$ and can consistently explain the DM relic density measurements. In contrast with most of the existing literature, we have found a reasonable DM production from the annihilation processes. After numerically studying the DM production, we show the dependence of the DM relic density as a function of its relevant free parameters. We use these results to obtain the parameter space regions that are compatible with the DM relic density bound. Secondly, we study a two component DM scenario and emphasize that the current DM relic density bound can be satisfied for a wide range of parameter space.

Keywords: Cosmology of Theories beyond the SM, Neutrino Physics

ARXIV EPRINT: 1904.10015 


\section{Contents}

1 Introduction 1

$2 \quad B-L$ model with inverse seesaw scenario and neutrino masses $\quad 3$

3 Warm DM as FIMP $\quad 6$

4 Two component FIMP dark matter $\quad 13$

4.1 Region I: $M_{Z^{\prime}}>2 M_{\nu_{H}^{1}} \quad 14$

4.2 Region II: $M_{Z^{\prime}}<2 M_{\nu_{H}^{1}} \quad 16$

$\begin{array}{lll}5 & \text { Conclusion } & 17\end{array}$

$\begin{array}{ll}\text { A Analytical expression of the collision terms } & 18\end{array}$

\section{Introduction}

The standard model (SM) is a very successful theory in describing nature. The discovery of the last missing piece of the SM, viz., the Higgs boson, further increases its concreteness. In spite of its tremendous success, the SM can not explain a number of phenomena - two of the most important ones being the presence of dark matter (DM) and non-zero neutrino mass. Presence of DM in the universe is a very well established fact. The first indication of DM came from the observation of Galactic velocities within the Coma cluster by Fritz Zwicky in 1933 [1], followed by the observation of galaxy rotation curves by Vera Rubin in 1970 [2]. Subsequently, the observation of bullet cluster [3] firmly confirmed the presence of DM. Currently the best measurement of the amount of DM present in the universe comes from the Planck data [4],

$$
\Omega h^{2}=0.1199 \pm 0.0027 \text { at } 68 \% \mathrm{CL},
$$

where $h$ is the reduced Hubble parameter and of order unity. Unfortunately, the SM does not have any fundamental particle which can be a viable DM candidate. Therefore, to address the issue of DM from particle physics point of view, we need to extend the SM particle content and/or its gauge group. One of the most promising scenarios is to consider the DM candidate as a Weakly Interacting Massive Particle (WIMP) [5, 6], which is produced in the early universe through the thermal freeze-out mechanism $[5,6]$. However, WIMP type DM attracts stringent bounds from direct and indirect detection experiments [7-14]. In particular, a large portion of the parameter space in the spin independent/dependent WIMP-nucleon cross section and DM mass plane is ruled out by the direct detection (DD) bounds. Moreover, in near future with increasing sensitivity of the DD experiments [7-11], these bounds might touch the so-called neutrino floor $[15,16]$. In this work, we follow a non-thermal way of DM production, viz., via the freeze-in mechanism [17]. In this scenario, 
the DM is very feebly interacting with the other particles, and as a result never achieves thermal equilibrium in the early universe with the cosmic soup. Hence it is named Feebly Interacting Massive Particles (FIMPs). Due to their very feeble interactions, FIMPs easily escape the above mentioned DD bounds while satisfying the measured value for the DM relic density [17-25].

On the other hand, results of the neutrino oscillation experiments [26-36] have confirmed oscillations between neutrino flavours. Since neutrino flavour oscillations are a clear proof of the neutrinos being massive and mixed, the neutrino oscillation experiments contradict the SM which postulates that the neutrinos are massless. Consequently, in order to explain tiny neutrino masses, one has to extend the SM by adding new particles and/or additional gauge groups.

In the present work we explain the above two puzzles by extending the SM gauge group by a $\mathrm{U}(1)_{B-L}$ gauge symmetry as a simple (minimal) and well motivated extension of the SM, where $B$ is the baryon number and $L$ is the lepton number. In addition to the extra neutral gauge boson $Z^{\prime}$ associated with the $\mathrm{U}(1)_{B-L}$, an extra SM singlet scalar $\phi_{H}$ (charged under $\mathrm{U}(1)_{B-L}$ to break $B-L$ gauge symmetry spontaneously) is added in this simple extension, which leads to interesting signatures at the LHC [37-41]. Moreover, nine additional SM singlet fermions $\left(N_{R}^{i}\right.$ and $\left.S_{1,2}^{i}, i=1,2,3\right)$ are needed to explain the naturally ${ }^{1}$ small neutrino masses through the inverse seesaw mechanism [49-52]. These additional fermions are not only required to generate the tiny neutrino masses via the inverse seesaw mechanism but are also needed for the gauge anomaly cancellation. In such a framework, three of these SM singlet fermions, $S_{1}^{i}$, are completely decoupled due to the introduction of $\mathbb{Z}_{2}$ symmetry and have naturally small mass (of order keV) according to 't Hooft's naturalness criterion [53]. Therefore, the lightest one, $S_{1}^{1}$, will be a stable particle and hence a warm DM (WDM) candidate [54-57], as discussed in ref. [58]. Moreover, since these $\mathrm{keV}$ mass singlet fermions are odd under the $\mathbb{Z}_{2}$ symmetry, they have no mixing with the active neutrinos and consequently are safe from the bound imposed by the $\mathrm{x}$ ray observations [59]. In ref. [58], an extra moduli field was introduced to produce this $\mathrm{keV}$ WDM non-thermally to achieve the correct ballpark value of relic density consistent with the WMAP and Planck observations. In the current work, without introducing any extra field contrary to ref. [58], we successfully produce the keV WDM by the freeze-in mechanism through the decay and annihilation channels of $Z^{\prime}$. After explaining the keV FIMP WDM as a successful single component FIMP DM scenario to satisfy the correct value of the DM relic density, we study a two component FIMP DM as another possible scenario in the present model, where in addition to the FIMP WDM $S_{1}^{1}$, the lightest heavy right-handed neutrino $\nu_{H}^{1}$ can be a FIMP DM (with mass of order GeV) by tuning its corresponding Yukawa coupling to be very small [60,61]. The GeV scale FIMP DM can be produced through the decay and annihilation processes of both the extra neutral gauge boson $Z^{\prime}$ as well as the extra $B-L$ Higgs $h^{\prime}$, while the keV FIMP WDM is produced only through the decay and annihilation processes of $Z^{\prime}$.

\footnotetext{
${ }^{1}$ Here, "naturally" means the Dirac neutrino masses, $M_{D}$, have the same size as the Dirac masses of the SM fermions and, in contrary to the usual type-I seesaw mechanism [42-48], large Dirac neutrino Yukawa couplings, $\lambda_{d} \sim \mathcal{O}(0.1)$, with right-handed neutrino $\left(N_{R}^{i}\right)$ masses are of order TeV.
} 


\begin{tabular}{|c|c|c|c|c|c|c|c|c|c|c|}
\hline \multirow{2}{*}{$\begin{array}{c}\text { Gauge } \\
\text { Group }\end{array}$} & \multicolumn{4}{|c|}{ Baryon Fields } & \multicolumn{4}{c|}{ Lepton Fields } & \multicolumn{3}{c|}{ Scalar Fields } \\
\cline { 2 - 11 } & $Q_{L}^{i}=\left(u_{L}^{i}, d_{L}^{i}\right)^{T}$ & $u_{R}^{i}$ & $d_{R}^{i}$ & $L_{L}^{i}=\left(\nu_{L}^{i}, e_{L}^{i}\right)^{T}$ & $e_{R}^{i}$ & $N_{R}^{i}$ & $S_{1}^{i}$ & $S_{2}^{i}$ & $\phi_{h}$ & $\phi_{H}$ \\
\hline $\mathrm{SU}(2)_{L}$ & 2 & 1 & 1 & 2 & 1 & 1 & 1 & 1 & 2 & 1 \\
\hline $\mathrm{U}(1)_{Y}$ & $1 / 6$ & $2 / 3$ & $-1 / 3$ & $-1 / 2$ & -1 & 0 & 0 & 0 & $1 / 2$ & 0 \\
\hline $\mathrm{U}(1)_{B-L}$ & $1 / 3$ & $1 / 3$ & $1 / 3$ & -1 & -1 & -1 & -2 & 2 & 0 & -1 \\
\hline
\end{tabular}

Table 1. Complete particle spectrum and their corresponding charges under various gauge groups.

The rest of the paper is organized as follows. In section 2 we discuss the $B-L$ model with inverse seesaw mechanism and how the light neutrinos acquire their tiny masses. In section 3 we show that a $\mathrm{keV}$ sterile neutrino can be a WDM and produce the observed DM relic density as a single component FIMP DM. Section 4 is dedicated for studying two component FIMP type DM. Finally, our conclusions are given in section 5.

\section{$2 \quad B-L$ model with inverse seesaw scenario and neutrino masses}

The gauged $B-L$ extension of the SM (BLSM) is based on the gauge group $\mathrm{SU}(3)_{C} \times$ $\mathrm{SU}(2)_{L} \times \mathrm{U}(1)_{Y} \times \mathrm{U}(1)_{B-L}$. By imposing $\mathrm{U}(1)_{B-L}$, the gauge sector of the $\mathrm{SM}$ is extended to include a new neutral gauge boson $Z^{\prime}$ associated with the $B-L$ gauge symmetry. In addition, it has three SM singlet fermions $N_{R}^{i}$ (three right-handed neutrinos) with $B-L$ charge $=-1$ that arise as a result of anomaly cancellation conditions. Included also is an extra SM singlet scalar $\phi_{H}$ with $B-L$ charge $=-1$, while $\phi_{h}$ is the usual electroweak (EW) Higgs doublet. In order to satisfy the experimental measurements for the nonvanishing light neutrino masses with $\mathrm{TeV}$ scale right-handed $(\mathrm{RH})$ neutrino using type-I seesaw mechanism, a very small Dirac neutrino Yukawa couplings, $\lambda_{d} \lesssim \mathcal{O}\left(10^{-6}\right)$ must be assumed [42-48]. Therefore, the mixing angle between the left- and right-handed neutrinos is quite suppressed, as it is proportional to $\lambda_{d} \lesssim \mathcal{O}\left(10^{-6}\right)$. As a consequence of such small mixing angle, the interactions between the $\mathrm{RH}$ neutrinos and the SM particles are very suppressed, making it difficult to observe them at the LHC [37-41]. Thus, we generate neutrino masses using the so-called inverse seesaw mechanism [49-52] that can naturally accommodate light neutrino masses with $\mathrm{TeV}$ scale $\mathrm{RH}$ neutrinos and large Yukawa couplings. In addition to the particle content as mentioned above, the BLSM with Inverse Seesaw (BLSMIS) has three extra pairs of SM singlet fermions $\left(S_{1,2}^{i}, i=1,2,3\right)$ with $B-L$ charge $=\mp 2$, respectively. In table 1 , we show the complete particle spectrum for the BLSMIS model with their associated charges for different gauge groups. An additional discrete symmetry has been introduced, viz., $\mathbb{Z}_{2}$. All BLSMIS particles are even under this symmetry except $S_{1}$ which is odd. Due to this symmetry, terms like $N_{R}^{c} \phi_{H}^{\dagger} S_{1}$ and $S_{1} S_{2}$, that could spoil the usual inverse seesaw mechanism, are forbidden [49-52]. The complete Lagrangian for this model is given by

$$
\begin{aligned}
\mathcal{L}= & \mathcal{L}_{\mathrm{SM}}-\frac{1}{4} F_{\mu \nu}^{\prime} F^{\prime \mu \nu}+\left(D_{\mu} \phi_{H}\right)^{\dagger} D_{\mu} \phi_{H}+\frac{i}{2} \bar{N}_{R} \gamma^{\mu} D_{\mu} N_{R}+\frac{i}{2} \bar{S}_{1} \gamma^{\mu} D_{\mu} S_{1}+\frac{i}{2} \bar{S}_{2} \gamma^{\mu} D_{\mu} S_{2} \\
& -\mathcal{V}\left(\phi_{h}, \phi_{H}\right)-\left(\lambda_{d} \bar{L} \tilde{\phi}_{h} N_{R}+\lambda_{s} \bar{N}_{R}^{c} \phi_{H} S_{2}+\text { h.c. }\right),
\end{aligned}
$$


where $F_{\mu \nu}^{\prime}=\partial_{\mu} B_{\nu}^{\prime}-\partial_{\nu} B_{\mu}^{\prime}$ is the $\mathrm{U}(1)_{B-L}$ field strength, $D_{\mu}$ is the covariant derivative, $\tilde{\phi}_{h}=i \sigma_{2} \phi_{h}$ and the flavor indices are omitted for simplicity. The general structure of the covariant derivative $D_{\mu}$ in the present model takes the following form

$$
D_{\mu}=\partial_{\mu}-i g_{c} T^{\alpha} G_{\mu}^{\alpha}-i g \tau^{a} W_{\mu}^{a}-i g_{Y} Y B_{\mu}-i g^{\prime} Y_{B L} B_{\mu}^{\prime},
$$

where $\left(g_{c}, T^{\alpha}, G_{\mu}^{\alpha}\right)$ are the $\mathrm{SU}(3)_{C}$ gauge coupling, generator and the gauge field, respectively. Similarly, $\left(g, \tau^{a}, W_{\mu}^{a}\right),\left(g_{Y}, Y, B_{\mu}\right)$ and $\left(g^{\prime}, Y_{B L}, B_{\mu}^{\prime}\right)$ are the corresponding quantities for $\mathrm{SU}(2)_{L}, \mathrm{U}(1)_{Y}$ and $\mathrm{U}(1)_{B-L}$, respectively. It is worth mentioning that a kinetic mixing term $F_{\mu \nu}^{\prime} F^{\mu \nu}$ is allowed and it leads to a non-vanishing $Z-Z^{\prime}$ mixing angle, $\theta^{\prime}$ [62-64]. However, due to the stringent constraint from LEP experiments on the $Z-Z^{\prime}$ mixing angle $\left(\left|\theta^{\prime}\right| \lesssim 10^{-3}\right)[65-67]$, one may neglect this term. Finally, the potential $\mathcal{V}\left(\phi_{h}, \phi_{H}\right)$ is given by $[37,38]$

$$
\mathcal{V}\left(\phi_{h}, \phi_{H}\right)=\mu_{h}^{2} \phi_{h}^{\dagger} \phi_{h}+\mu_{H}^{2} \phi_{H}^{\dagger} \phi_{H}+\lambda_{h}\left(\phi_{h}^{\dagger} \phi_{h}\right)^{2}+\lambda_{H}\left(\phi_{H}^{\dagger} \phi_{H}\right)^{2}+\lambda_{h H}\left(\phi_{h}^{\dagger} \phi_{h}\right)\left(\phi_{H}^{\dagger} \phi_{H}\right),
$$

where the potential $\mathcal{V}\left(\phi_{h}, \phi_{H}\right)$ will be bounded from below when the following inequalities are satisfied simultaneously

$$
\mu_{h}^{2}<0, \quad \mu_{H}^{2}<0, \quad \lambda_{h} \geq 0, \quad \lambda_{H} \geq 0 \text { and } \lambda_{h H} \geq-2 \sqrt{\lambda_{h} \lambda_{H}} .
$$

Here, both the scalars $\phi_{H}$ and $\phi_{h}$ acquire their non-zero vacuum expectation values (VEVs), therefore, the $B-L$ and the EW symmetries are broken spontaneously and the SM Higgs doublet $\phi_{h}$ and the $B-L$ singlet $\phi_{H}$ take the following form:

$$
\phi_{h}=\left(\begin{array}{c}
0 \\
\frac{v+h}{\sqrt{2}}
\end{array}\right), \quad \phi_{H}=\frac{v^{\prime}+H}{\sqrt{2}},
$$

where $v \simeq 246 \mathrm{GeV}$ is the EW symmetry breaking scale and $v^{\prime}$ is the scale of $B-L$ symmetry breaking which is, in general, unknown and ranging from $\mathrm{TeV}$ to much higher scales. After breaking the $B-L$ and the EW symmetries spontaneously, the extra neutral gauge boson $Z^{\prime}$ acquires its mass $\left(M_{Z^{\prime}}=g^{\prime} v^{\prime}\right)[37,38],{ }^{2}$ and the neutrino Yukawa interaction terms in eq. (2.1) and in addition a very small Majorana mass $\mu_{S}$ for $S_{1,2}$ lead to the following neutrino mass terms ${ }^{3}$

$$
\mathcal{L}_{m}^{\nu}=\mu_{S}\left(\bar{S}_{1}^{c} S_{1}+\bar{S}_{2}^{c} S_{2}\right)+\left(M_{D} \bar{\nu}_{L} N_{R}+M_{N} \bar{N}_{R}^{c} S_{2}+\text { h.c. }\right),
$$

where $M_{D}=\lambda_{d} v / \sqrt{2}$ and $M_{N}=\lambda_{s} v^{\prime} / \sqrt{2}$. Therefore, the neutrino mass matrix in the basis $\left(\nu_{L}^{c}, N_{R}, S_{2}, S_{1}\right)$ can be written as

$$
\mathcal{M}_{\nu}=\left(\begin{array}{cccc}
0 & M_{D} & 0 & 0 \\
M_{D}^{T} & 0 & M_{N} & 0 \\
0 & M_{N}^{T} & \mu_{S} & 0 \\
0 & 0 & 0 & \mu_{S}
\end{array}\right) .
$$

\footnotetext{
${ }^{2}$ The experimental search for $Z^{\prime}$, by LEP II $[68,69]$, leads to another constraint: $M_{Z^{\prime}} / g^{\prime} \gtrsim 7 \mathrm{TeV}$. This constraint will easily be satisfied due to a smallness of $g^{\prime}$ which is required by the freeze-in scenario [17].

${ }^{3} \mu_{S}$ is naturally small due to 't Hooft's naturalness criterion [53], for simplicity we assume $S_{1}$ and $S_{2}$ have the same small Majorana mass $\left(\mu_{S}\right)$, and the generation of such small $\mu_{S}$ from non-renormalizable terms has been discussed in [70] and radiatively in [71].
} 
It is clearly seen that $S_{1}$ is completely decoupled and has no mixing with active neutrinos. It only interacts with the neutral gauge boson $Z^{\prime}$ with a coupling $g_{Z^{\prime} S_{1} S_{1}}=g^{\prime}$. Therefore, $S_{1}$ is free from cosmological and astrophysical constraints coming from active-sterile mixing [59]. Thus its mass is given as,

$$
M_{S_{1}}=\mu_{S}
$$

After diagonalising the upper left $3 \times 3$ submatrix of the neutrino mass matrix $\mathcal{M}_{\nu}$, the light and heavy neutrino masses, respectively, are given by

$$
\begin{aligned}
M_{\nu_{l}} & \simeq M_{D} M_{N}^{-1} \mu_{S}\left(M_{N}^{T}\right)^{-1} M_{D}^{T}, \\
M_{\nu_{H, H^{\prime}}}^{2} & \simeq M_{N}^{2}+M_{D}^{2} \mp \frac{1}{2} \frac{M_{N}^{2} \mu_{S}}{M_{N}^{2}+M_{D}^{2}},
\end{aligned}
$$

where $\mu_{S} \ll M_{D}, M_{N}$ is assumed. One can naturally obtain eV scale light neutrino masses with $\mu_{S}$ of order keV and $M_{N}$ of order TeV, keeping Yukawa coupling $\lambda_{d}$ of order one. Such large couplings between the heavy $\mathrm{RH}$ neutrinos and the SM particles leads to interesting implications and enhances the accessibility of TeV scale $B-L$ at the LHC [72-74].

Recall that due to the added $\mathbb{Z}_{2}$ symmetry, $S_{1}$ is completely decoupled. Hence the lightest fermionic singlet, $S_{1}^{1}$, is a stable particle and hence a DM candidate. Since its mass $\left(=\mu_{S}\right)$ is of order $\mathrm{keV}$, hence $S_{1}^{1}$ is a warm DM (WDM) candidate [58]. ${ }^{4}$ Moreover, one can easily make the lightest heavy $\mathrm{RH}$ neutrino, $\nu_{H}^{1}$, stable or long-lived by taking the corresponding Yukawa coupling to be very small $\lesssim 3 \times 10^{-26}\left(\mathrm{GeV} / M_{N}\right)^{1 / 2}[60,61]$. Thus, from here onwards we focus on the two component DM scenario, where, one of them is GeV scale DM, $\nu_{H}^{1}$, and the other is keV scale WDM, $S_{1}^{1}$.

It is important to note that due to the mixing term in the potential $\mathcal{V}\left(\phi_{h}, \phi_{H}\right)$, the squared mass matrix of the neutral Higgs bosons in the basis $(h, H)$ is non-diagonal and takes the following form:

$$
\mathcal{M}_{\mathrm{scalar}}^{2}=\left(\begin{array}{cc}
2 \lambda_{h} v^{2} & \lambda_{h H} v^{\prime} v \\
\lambda_{h H} v^{\prime} v & 2 \lambda_{H} v^{\prime 2}
\end{array}\right)
$$

Rotating this matrix into the basis $\left(h_{1}, h_{2}\right)$ which is defined as follows

$$
\begin{aligned}
& h_{1}=h \cos \alpha+H \sin \alpha, \\
& h_{2}=-h \sin \alpha+H \cos \alpha,
\end{aligned}
$$

where the mixing angle $\alpha$ takes the following form:

$$
\tan 2 \alpha=\frac{\lambda_{h H} v^{\prime} v}{\lambda_{H} v^{\prime 2}-\lambda_{h} v^{2}}
$$

\footnotetext{
${ }^{4}$ The contribution of the new light degrees of freedom $\left(S_{1}^{i}\right)$ to the number of effective neutrino species, $N_{\text {eff }}$, has been checked using eq. (5) in ref. [75] to calculate extra effective neutrino species, $\Delta N_{\text {eff }}$, and found it to be negligible.
} 
Therefore, the masses of these two physical Higgs scalars $\left(h_{1}, h_{2}\right)$ are given by ${ }^{5}$

$$
M_{h_{1,2}}^{2}=\lambda_{h} v^{2}+\lambda_{H} v^{\prime 2} \mp \sqrt{\left(\lambda_{H} v^{\prime 2}-\lambda_{h} v^{2}\right)^{2}+\left(\lambda_{h H} v v^{\prime}\right)^{2}} .
$$

The quartic couplings $\lambda$ 's can be written in terms of the physical masses $M_{h_{1,2}}$ as follows [78]

$$
\begin{aligned}
\lambda_{h} & =\frac{M_{h_{1}}^{2}+M_{h_{2}}^{2}-\left(M_{h_{2}}^{2}-M_{h_{1}}^{2}\right) \cos 2 \alpha}{4 v^{2}}, \\
\lambda_{H} & =\frac{M_{h_{1}}^{2}+M_{h_{2}}^{2}+\left(M_{h_{2}}^{2}-M_{h_{1}}^{2}\right) \cos 2 \alpha}{4 v^{\prime 2}}, \\
\lambda_{h H} & =\frac{\left(M_{h_{2}}^{2}-M_{h_{1}}^{2}\right) \sin 2 \alpha}{2 v v^{\prime}} .
\end{aligned}
$$

We have used SARAH [79-81] to implement the BLSMIS and the relevant masses, couplings and decay widths have been calculated using SPheno [82].

\section{Warm DM as FIMP}

As mentioned earlier, $S_{1}^{1}$ is a WDM candidate with mass in the few $\mathrm{keV}$ range [83-85]. We next study in detail the production of this keV DM via the freeze-in mechanism. Here $S_{1}^{1}$ is produced solely from its coupling with the extra $\mathrm{U}(1)_{B-L}$ gauge boson $Z^{\prime}$, as mentioned in the previous section. The corresponding gauge coupling $g^{\prime}$ is taken to be extremely feeble $\sim \mathcal{O}\left(10^{-10}\right)$ with the result that $S_{1}^{1}$ is never in thermal equilibrium with the cosmic soup. Due to this small $B-L$ gauge coupling, the corresponding gauge boson $Z^{\prime}$ also interacts very feebly with the cosmic soup and never attains thermal equilibrium [86],

$$
\frac{\Gamma_{Z^{\prime}}}{H\left(T=M_{Z^{\prime}}\right)}<1
$$

where $\Gamma_{Z^{\prime}}$ is the total decay width of $Z^{\prime}$ and $H$ is the Hubble parameter. Therefore, we first determine the distribution function for $Z^{\prime}{ }^{6}$ The general formalism to determine the distribution function of any particle (say $f$ ) is to solve the following Boltzmann equation:

$$
\hat{L}[f]=\mathcal{C}[f],
$$

where $\hat{L}$ is the Lioville's operator and $\mathcal{C}[f]$ is known as the collision term of $f$. If we consider an isotropic and homogeneous universe, then, using the Friedman-RobertsonWalker metric, the Lioville's operator takes the following form:

$$
\hat{L}=\frac{\partial}{\partial t}-H p \frac{\partial}{\partial p}
$$

\footnotetext{
${ }^{5}$ Hereafter, the physical state $h_{1}$ refers to the SM-like Higgs boson and its mass $M_{h_{1}}$ is fixed at $125.5 \mathrm{GeV}$ to agree with the LHC measurements [76, 77]. Also, according to the measured values of Higgs boson signal strengths for its various decay modes, the mixing angle $\alpha$ should be very small, thus we have fixed it at $0.01 \mathrm{rad}$.

${ }^{6}$ As $Z^{\prime}$ is not in thermal equilibrium (due to very small value of $g^{\prime}$ ), one can not assume a MaxwellBoltzmann distribution function for $Z^{\prime}$. Therefore, the $Z^{\prime}$ distribution can be found by solving eq. (3.2).
} 
where $p$ is the absolute value of the particle's three momentum, $|\vec{p}|$. Following [18], we perform a transformation of variables, $(p, t) \rightarrow\left(\xi_{p}, z\right)$, in the following way:

$$
z=\frac{M_{\mathrm{sc}}}{T}, \quad \xi_{p}=\left[\frac{g_{s}\left(T_{0}\right)}{g_{s}(T)}\right]^{1 / 3} \frac{p}{T},
$$

where $g_{s}(T)$ is the effective entropy degrees of freedom (d.o.f) at temperature $T, M_{\mathrm{sc}}$ is an arbitrary mass scale and hereafter we take it equal to the SM-like Higgs mass $\left(M_{\mathrm{sc}}=M_{h_{1}}=125.5 \mathrm{GeV}\right)$ and $T_{0}$ is the initial temperature at which the DM relic density is taken to be zero. Therefore, using the following time-temperature relation,

$$
\frac{d T}{d t}=-H T\left(1+\frac{T g_{s}^{\prime}(T)}{3 g_{s}(T)}\right)^{-1}
$$

the Lioville's operator defined in eq. (3.3) can be simply written as

$$
\hat{L}=z H\left(1+\frac{T g_{s}^{\prime}}{3 g_{s}}\right)^{-1} \frac{\partial}{\partial z},
$$

where $g_{s}^{\prime}(T)$ is the derivative of $g_{s}(T)$ with respect to the temperature $T$.

Taking only the decay term for the $Z^{\prime}$ production, ${ }^{7}$ the Boltzmann equation of the distribution function of $Z^{\prime}$ is given by

$$
\hat{L} f_{Z^{\prime}}=\sum_{i=1,2} \mathcal{C}^{h_{i} \rightarrow Z^{\prime} Z^{\prime}}+\mathcal{C}^{Z^{\prime} \rightarrow \text { all }}
$$

where $f_{Z^{\prime}}$ is the distribution function of $Z^{\prime}, \mathcal{C}^{h_{i} \rightarrow Z^{\prime} Z^{\prime}}$ is the collision term of $Z^{\prime}$ production from the decays of scalars $h_{1,2}$ and $\mathcal{C}^{Z^{\prime} \rightarrow \text { all }}$ is $Z^{\prime}$ decay collision term due to all its possible decay channels. The expression of these collision terms are given in the appendix A. Once we get the distribution function of $Z^{\prime}$ by solving eq. (3.7), we then can determine its co-moving number density by using the following relation:

$$
Y_{Z^{\prime}}=\frac{n_{Z^{\prime}}(z)}{s}=\frac{45 g}{4 \pi^{4} g_{s}\left(M_{\mathrm{sc}} / z_{0}\right)} \int_{0}^{\infty} d \xi_{p} \xi_{p}^{2} f_{Z^{\prime}}\left(\xi_{p}, z\right),
$$

where $n_{Z^{\prime}}$ is the $Z^{\prime}$ number density, $g$ is the internal d.o.f of $Z^{\prime}$ and the universe entropy density $s$ is given by $s=\left(2 \pi^{2} / 45\right) T^{3} g_{s}(T)$ [87].

From eq. (3.8), one can note that the co-moving number density of $Z^{\prime}$ is directly proportional to the integrated $\xi_{p}^{2} f_{Z^{\prime}}$, i.e., larger the area under a $\xi_{p}^{2} f_{Z^{\prime}}$ curve, larger is the $Z^{\prime}$ abundance. In figure 1 , we show the variation of $\xi_{p}^{2} f_{Z^{\prime}}$ with respect to the dimensionless parameter $\xi_{p}$ for different values of $z\left(=M_{\mathrm{sc}} / T\right)$. As shown in the figure, areas under the curves corresponding to $z=0.02$ and 20.0 are different because for higher $z=20.0$ (i.e., lower temperature $T$ of the universe), $Z^{\prime}$ gets more time to be produced and it

\footnotetext{
${ }^{7}$ In principle, the collision term for annihilation diagrams should also be considered but in this class of models those annihilation diagrams have subleading contribution [19], hence we have not taken into account those effects and for simplicity we consider only the decay of $h_{1,2}$ as the $Z^{\prime}$ production mechanism. Moreover, $h_{1,2}$ are in thermal equilibrium, and consequently the usual equilibrium Boltzmann distribution function has been assumed for them [18].
} 


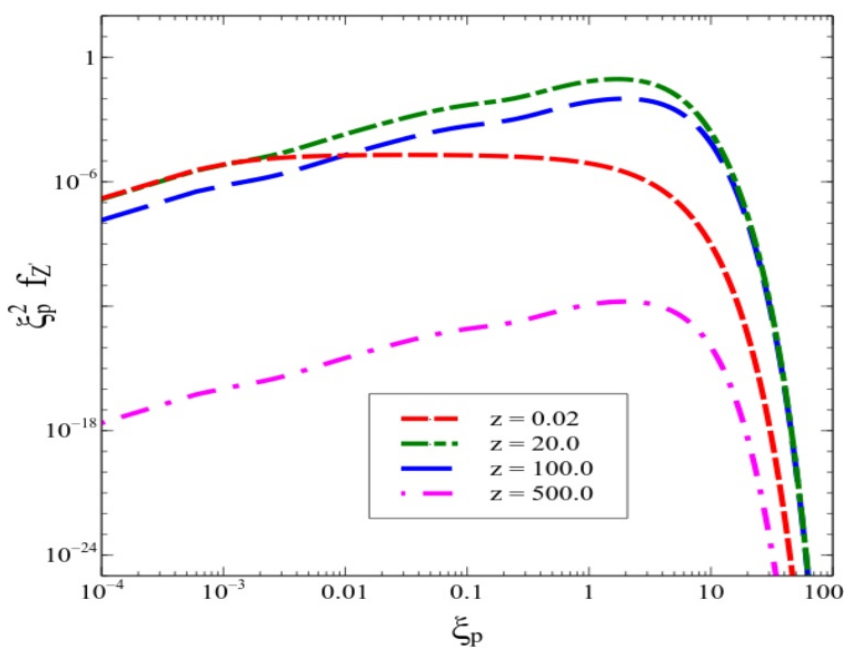

Figure 1. $Z^{\prime}$ distribution function versus the dimensionless parameter $\xi_{p}$. Here, the relevant BLSMIS parameters are fixed as follows: $g^{\prime}=12.5 \times 10^{-10}, M_{Z^{\prime}}=10 \mathrm{GeV}, M_{h_{2}}=1 \mathrm{TeV}, M_{S_{1}^{1}}=$ $10 \mathrm{keV}$, and $\alpha=0.01 \mathrm{rad}$.

then subsequently decays into WDM and the SM fermions. But as $z$ is increased further (presently $z=100.0$ ), $Z^{\prime}$ starts decaying significantly and its abundance gets depleted and the area under the curve for $z=100$ is smaller than for $z=20.0$, as seen in figure 1. For still higher values of $z(z=500.0), Z^{\prime}$ abundance decreases further due to decay. Thus, as $z \rightarrow \infty, Z^{\prime}$ will gradually decay to DM and its abundance eventually goes to zero.

Once the distribution function of $Z^{\prime}$ is computed, we can describe the production of the keV DM $S_{1}^{1}$. In the present scenario, the keV DM $S_{1}^{1}$ can be produced from the decay of $Z^{\prime}, Z^{\prime} \rightarrow S_{1}^{1} S_{1}^{1}$ (decay contribution), and from the annihilation processes, $f \bar{f} \rightarrow S_{1}^{1} S_{1}^{1}$ mediated by $Z^{\prime}$, where $f=l, q, \nu_{l}$. The annihilation contribution has been calculated by using micrOMEGAs [88]. To determine the co-moving number density of the WDM $S_{1}^{1}$, we solve the following Boltzmann equation,

$$
\begin{aligned}
\frac{d Y_{S_{1}^{1}}}{d z}= & \frac{2 M_{\mathrm{Pl}} z \sqrt{g_{\star}}}{1.66 M_{\mathrm{sc}}^{2} g_{s}}\left\langle\Gamma_{Z^{\prime} \rightarrow S_{1}^{1} S_{1}^{1}}\right\rangle_{\mathrm{NTH}}\left(Y_{Z^{\prime}}-Y_{S_{1}^{1}}\right) \\
& +\frac{4 \pi^{2}}{45} \frac{M_{\mathrm{Pl}} M_{\mathrm{sc}} \sqrt{g_{\star}}}{1.66 z^{2}} \sum_{f}\left\langle\sigma v_{f \bar{f} \rightarrow S_{1}^{1} S_{1}^{1}}\right\rangle\left[\left(Y_{f}^{\mathrm{eq}}\right)^{2}-Y_{S_{1}^{1}}^{2}\right],
\end{aligned}
$$

where $M_{\mathrm{Pl}}=1.22 \times 10^{19} \mathrm{GeV}$ is the Planck mass, $\sqrt{g_{\star}}=\frac{g_{s}(z)}{\sqrt{g_{\rho}(z)}}\left(1-\frac{1}{3} \frac{\mathrm{d} \ln g_{s}(z)}{\mathrm{d} \ln z}\right)$, where $g_{\rho}$ is the effective energy degrees of freedom [5, 89], and the non-thermal average of $Z^{\prime}$ decay width is defined by

$$
\left\langle\Gamma_{Z^{\prime} \rightarrow S_{1}^{1} S_{1}^{1}}\right\rangle_{\mathrm{NTH}}=M_{Z^{\prime}} \Gamma_{Z^{\prime} \rightarrow S_{1}^{1} S_{1}^{1}} \frac{\int_{0}^{\infty} d \xi_{p} \xi_{p} f_{Z^{\prime}}\left(\xi_{p}, z\right)\left(\left[\mathcal{B}(z) \frac{M_{\mathrm{sc}}}{z}\right]^{2}+\left[\frac{M_{Z^{\prime}}}{\xi_{p}}\right]^{2}\right)^{-1 / 2}}{\int_{0}^{\infty} d \xi_{p} \xi_{p}^{2} f_{Z^{\prime}}\left(\xi_{p}, z\right)}
$$



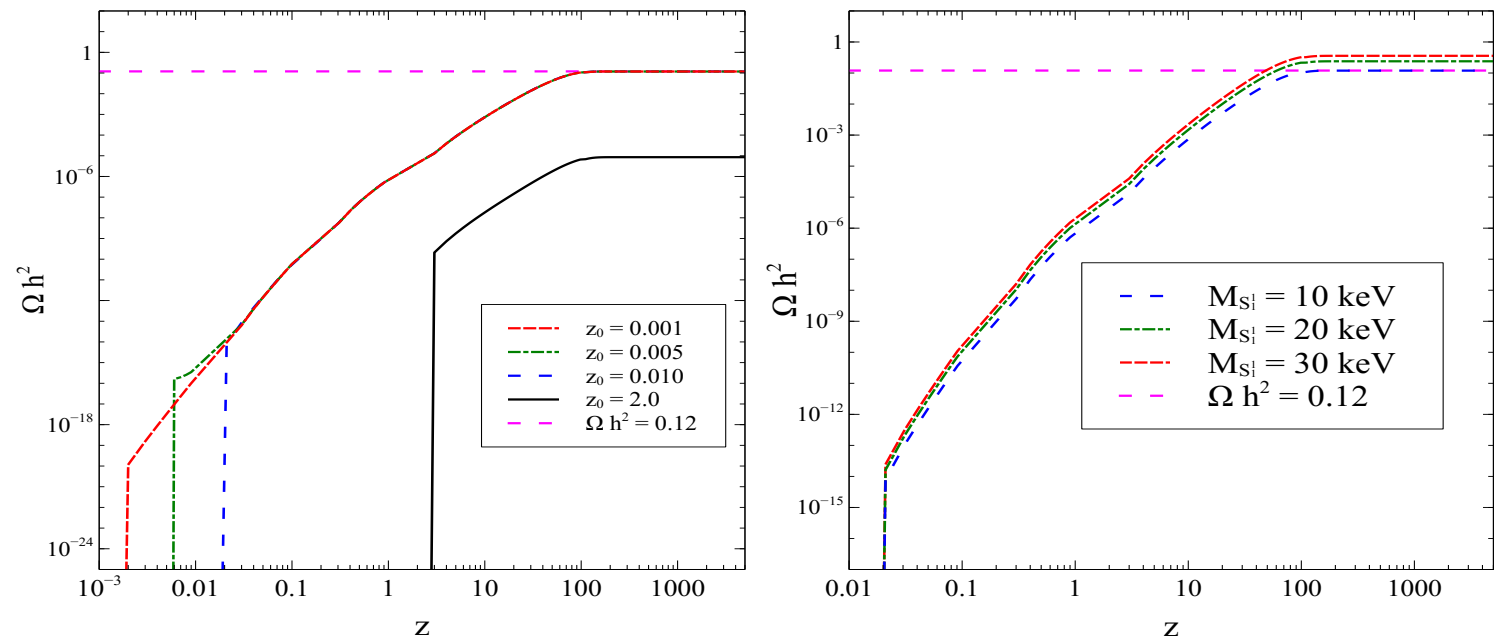

Figure 2. Variation of DM relic density, for the decay contribution only, as a function of $z$ for different values of $z_{0}$ (left), where $M_{S_{1}^{1}}=10 \mathrm{keV}$ and different values of the WDM mass $M_{S_{1}^{1}}$ (right), where $z_{0}=0.01$. Here $g^{\prime}=12.5 \times 10^{-10}, M_{Z^{\prime}}=10 \mathrm{GeV}, M_{h_{2}}=1 \mathrm{TeV}$, and $\alpha=0.01 \mathrm{rad}$.

where

$$
\mathcal{B}(z)=\left[\frac{g_{s}\left(M_{\mathrm{sc}} / z\right)}{g_{s}\left(M_{\mathrm{sc}} / z_{0}\right)}\right]^{1 / 3}
$$

The expressions of a thermal average annihilation cross section $\left\langle\sigma v_{f \bar{f} \rightarrow S_{1}^{1} S_{1}^{1}}\right\rangle$ and an equilibrium co-moving number density of $f\left(Y_{f}^{\mathrm{eq}}\right)$, appearing in eq. (3.9), are given respectively by $[5,89]$

$$
\begin{aligned}
\left\langle\sigma v_{f \bar{f} \rightarrow S_{1}^{1} S_{1}^{1}}\right\rangle & =\frac{z}{8 M_{f}^{4} M_{\mathrm{sc}} K_{2}\left(z M_{f} / M_{\mathrm{sc}}\right)^{2}} \int_{4 M_{f}^{2}}^{\infty} \sigma_{f \bar{f} \rightarrow S_{1}^{1} S_{1}^{1}}\left(s-4 M_{f}^{2}\right) \sqrt{s} K_{1}\left(\frac{z \sqrt{s}}{M_{\mathrm{sc}}}\right) d s, \\
Y_{f}^{\mathrm{eq}} & =\frac{45 g_{f}}{4 \pi^{4}}\left(\frac{z M_{f}}{M_{\mathrm{sc}}}\right)^{2} \frac{K_{2}\left(z M_{f} / M_{\mathrm{sc}}\right)}{g_{s}\left(M_{\mathrm{sc}} / z\right)},
\end{aligned}
$$

where $g_{f}$ is the internal d.o.f of $f, K_{1}(z)$ and $K_{2}(z)$ are the Bessel function for first and second kind, respectively, and $\sigma_{f \bar{f} \rightarrow S_{1}^{1} S_{1}^{1}}$ is given in ref. [90]. Solving the Boltzmann equation given by eq. (3.9) gives us the co-moving number density $Y_{S_{1}^{1}}$. The corresponding relic density of the WDM $S_{1}^{1}$ can be calculated by using the following formula [89],

$$
\Omega h^{2} \simeq 2.755 \times 10^{8}\left(\frac{M_{S_{1}^{1}}}{\mathrm{GeV}}\right) Y_{S_{1}^{1}}(\infty) .
$$

In order to understand the relative contribution of the decay and annihilation channels we will first consider them one at a time and solve the Boltzmann equation to get the relic density. We start with taking only the decay contribution and show in the left and right panels of figure 2 the variation of DM relic density as a function of $z$, for different values of the initial temperature $T_{0}\left(=M_{\mathrm{sc}} / z_{0}\right)$ and different values of the WDM mass $M_{S_{1}^{1}}$, respectively. The horizontal magenta dashed line refers to the DM relic density 

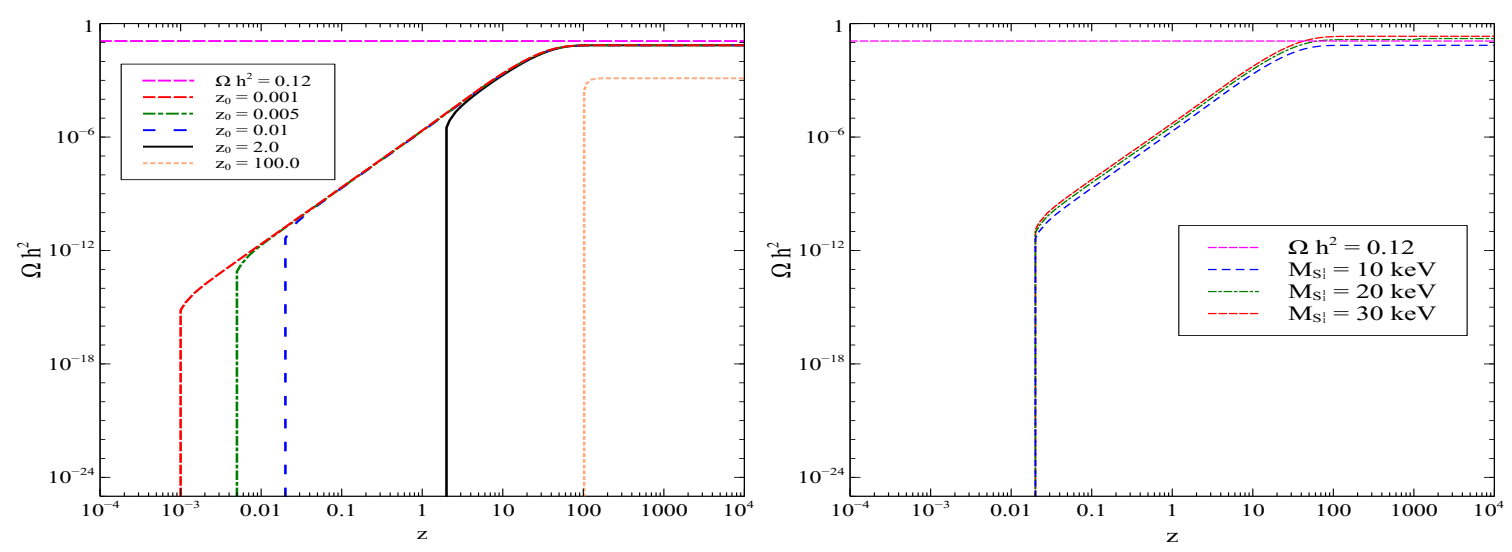

Figure 3. Variation of DM relic density, for the annihilation contribution only, as a function of $z$ for different values of $z_{0}$ (left), where $M_{S_{1}^{1}}=10 \mathrm{keV}$ and different values of the WDM mass $M_{S_{1}^{1}}$ (right), where $z_{0}=0.01$. Here, $g^{\prime}=12.5 \times 10^{-10}, M_{Z^{\prime}}=10 \mathrm{GeV}, M_{h_{2}}=1 \mathrm{TeV}$, and $\alpha=0.01 \mathrm{rad}$.

measurement $\left(\Omega h^{2} \simeq 0.12\right)$ [4]. In the left panel, as long as $T_{0}$ is greater than the mass of the mother particles $\left(h_{1,2}\right)$ in $h_{1,2} \rightarrow Z^{\prime} Z^{\prime}$ decay channels, the final DM relic density is insensitive to $T_{0}$, as seen for the $z_{0}=0.001,0.005,0.01$ cases. However, once $T_{0}$ drops below the mass of the mother particles (presently $z_{0}=2$ case), $Z^{\prime}$ production gets Boltzmann suppressed and consequently DM relic density is suppressed. In the right panel we show the dependence of the DM relic density on its mass $\left(M_{S_{1}^{1}}\right)$ for $z_{0}=0.01$. It is clear that the relic density increases with the DM mass, as expected from eq. (3.14).

For the annihilation contribution, $f \bar{f} \rightarrow S_{1}^{1} S_{1}^{1}$, there are two relevant regimes are as follows. (i) The on-shell regime, where $2 M_{S_{1}^{1}}<M_{Z^{\prime}}<T_{0}$, in which $Y_{S_{1}^{1}}\left(\propto g^{\prime 4} / \Gamma_{Z^{\prime}}\right)$ does not depend on $T_{0}$ and $(i i)$ The EFT regime, where $2 M_{S_{1}^{1}}<T_{0}<M_{Z^{\prime}}$, in which $Y_{S_{1}^{1}}\left(\propto g^{\prime 4} T_{0}^{3} / M_{Z^{\prime}}^{4}\right)$ depends on $T_{0}$. In the left panel of figure 3 we see that as long as $T_{0}$ is greater than $M_{Z^{\prime}}$, the final DM relic density is insensitive to $T_{0}$ (on-shell regime), as seen for the $z_{0}=0.001,0.005,0.01,2.0$ cases. Once $T_{0}$ drops below $M_{Z^{\prime}}$ (presently $z_{0}=100.0$ case), then $S_{1}^{1}$ production gets the suppressed by a factor $T_{0}^{3} / M_{Z^{\prime}}^{4}$ (EFT regime). In the right panel of figure 3 , we show the dependence of the DM relic density on its mass $\left(M_{S_{1}^{1}}\right)$ for $z_{0}=0.01$ (on-shell regime). It is clear that the relic density increases with the DM mass, as expected from eq. (3.14).

For the decay contribution $\left(Z^{\prime} \rightarrow S_{1}^{1} S_{1}^{1}\right)$, we show in figure 4 the variation of the comoving number density of $Z^{\prime}$ (left panel) and the co-moving number density of $S_{1}^{1}$ with $z$, for different values of $B-L$ gauge coupling $g^{\prime}$ (left panel) and $M_{Z^{\prime}}$ (right panel). Since $Z^{\prime}$ production is proportional to the $B-L$ gauge coupling $g^{\prime}$, larger $g^{\prime}$ results in larger $Z^{\prime}$ production and consequently a larger production of DM, as seen in the left panel of this figure. Note also that $Z^{\prime} \rightarrow S_{1}^{1} S_{1}^{1}$ decay rate is directly proportional to $g^{\prime}$, and hence increasing $g^{\prime}$ increases the decay rate of $Z^{\prime}$ and hence the abundance of $S_{1}^{1}$. Therefore, it is clear that for higher values of $g^{\prime}$ the $Z^{\prime}$ co-moving number density plateau starts bending at smaller values of $z$. On the other hand, in the right panel of figure 4, we see that by increasing $M_{Z^{\prime}}$ exactly opposite behavior appears for $Z^{\prime}$ production while similar behavior 

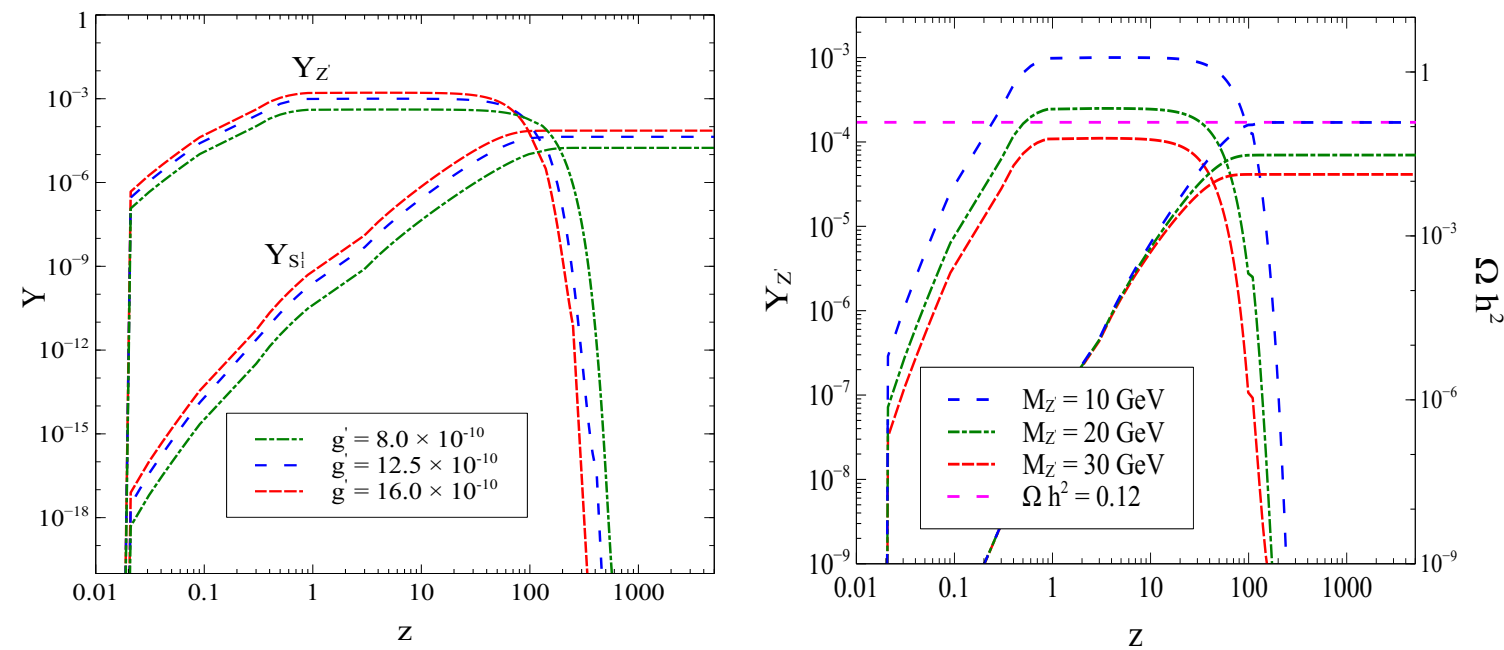

Figure 4. Variation of the co-moving number density of $Z^{\prime}$ and the WDM $S_{1}^{1}$ (for the decay contribution) as a function of $z$ for different values of $B-L$ gauge coupling $g^{\prime}$ (left) where $M_{Z^{\prime}}=$ $10 \mathrm{GeV}$ and $M_{Z^{\prime}}$ (right), where $g^{\prime}=12.5 \times 10^{-10}$. Here, $z_{0}=0.01, M_{S_{1}^{1}}=10 \mathrm{keV}, M_{h_{2}}=1 \mathrm{TeV}$, and $\alpha=0.01 \mathrm{rad}$.
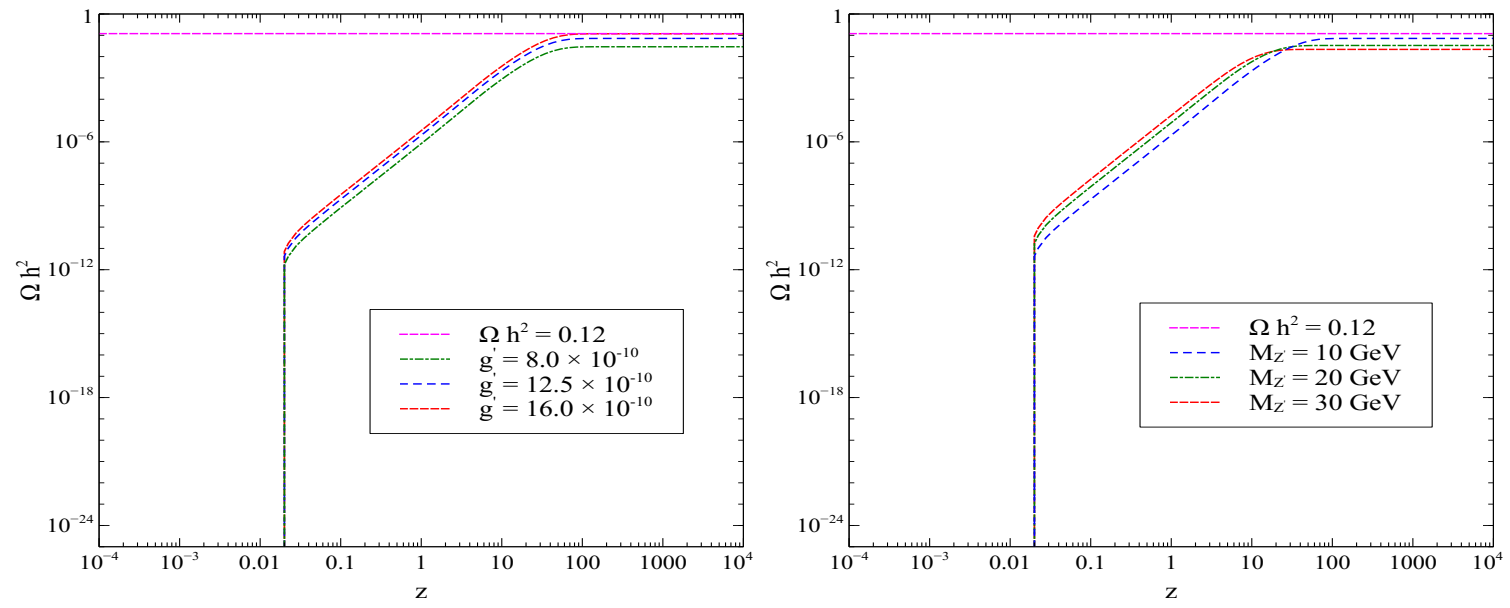

Figure 5. Variation of DM relic density, for the annihilation contribution, as a function of $z$ for different values of $B-L$ gauge coupling $g^{\prime}$ (left) where $M_{Z^{\prime}}=10 \mathrm{GeV}$ and $M_{Z^{\prime}}$ (right), where $g^{\prime}=12.5 \times 10^{-10}$. Here, $z_{0}=0.01, M_{S_{1}^{1}}=10 \mathrm{keV}, M_{h_{2}}=1 \mathrm{TeV}$, and $\alpha=0.01 \mathrm{rad}$.

happens for its decay. As mentioned, $Z^{\prime}$ production mainly happens through decay of the Higgs bosons $\left(h_{1,2}\right)$ and those decay modes $\left(\Gamma_{h_{i} \rightarrow Z^{\prime} Z^{\prime}}\right)$ are proportional to $M_{h_{i}}^{3} / M_{Z^{\prime}}^{2}$ [see eq. (A.6)]. Therefore, increasing $M_{Z^{\prime}}$ reduces the production rate of $Z^{\prime}$ as $1 / M_{Z^{\prime}}^{2}$. However, its decay width is simply proportional to its mass $M_{Z^{\prime}}$ [see eq. (A.3)] and so increasing $M_{Z^{\prime}}$ results in faster decay of $Z^{\prime}$. In figure 5 we show similar plots for the annihilation contribution. This figure shows features similar to figure 4 .

In figure 6 , we show the total relic density (blue dashed-dotted line) as well as the relative contributions of the two different types of WDM production processes, decay (red 


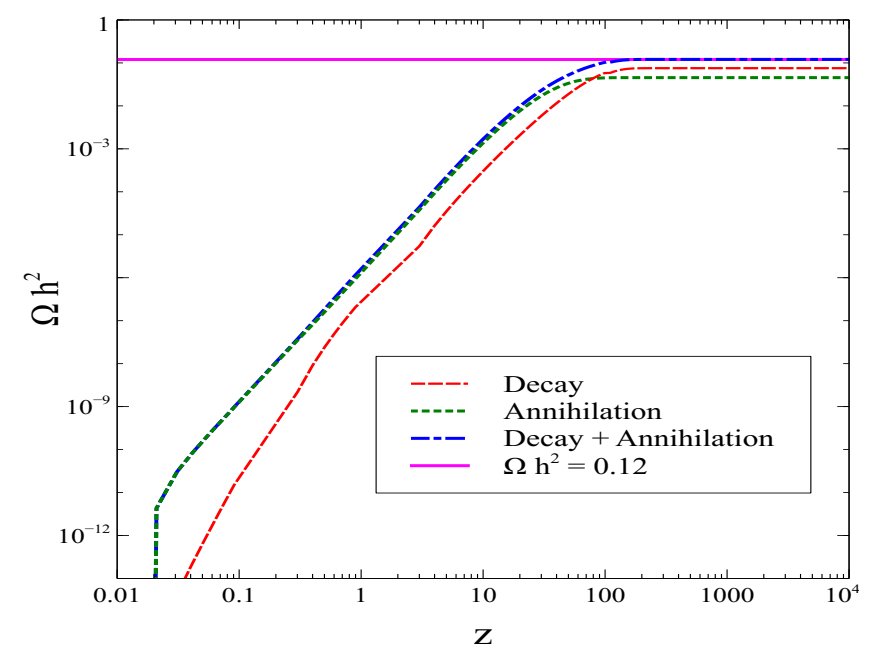

Figure 6. Variation of relic density contributions of the single DM component scenario as a function of $z$. Here, $M_{S_{1}^{1}}=10 \mathrm{keV}, M_{Z^{\prime}}=10 \mathrm{GeV}, g^{\prime}=1.0 \times 10^{-9}, M_{h_{2}}=1 \mathrm{TeV}, z_{0}=0.01$, and $\alpha=0.01 \mathrm{rad}$.

dashed line) and annihilation (green dotted line). Here, for a suitably selected set of model parameters $\left(M_{S_{1}^{1}}=10 \mathrm{keV}, M_{Z^{\prime}}=10 \mathrm{GeV}, g^{\prime}=1.0 \times 10^{-9}, M_{h_{2}}=1 \mathrm{TeV}, z_{0}=0.01\right.$, and $\alpha=0.01 \mathrm{rad})$, the total WDM relic density equals the observed relic density $\left(\Omega h^{2} \simeq 0.12\right)$ at the present epoch, where decay contributes $\sim 62 \%$ of the WDM relic density while the rest comes from annihilation. It is worth mentioning that initially for $z \lesssim 100$, WDM is dominantly produced from the annihilation processes this is because of all ingoing particles are already in the cosmic soup, while for $z \gtrsim 100$, the decay process starts dominating, as seen in figure 4.

Variation of total WDM relic density $\left(\Omega h^{2}\right)$ as a function of the gauge coupling $g^{\prime}$ can be seen in figure 7, where the BLSMIS points have been generated over the following ranges of its fundamental parameters: $1 \leq M_{S_{1}^{1}} \leq 10 \mathrm{keV}, 1 \leq M_{Z^{\prime}} \leq 100 \mathrm{GeV}, 10^{-12} \leq g^{\prime} \leq 10^{-8}$, $M_{h_{2}}=1 \mathrm{TeV}, z_{0}=0.01$, and $\alpha=0.01 \mathrm{rad}$. From the left panel it is clear that $\Omega h^{2}$ is inversely proportional to $M_{Z^{\prime}}$ (which is represented by the color bar). More explicitly, for a fixed $g^{\prime}$ value, larger $\Omega h^{2}$ values correspond to smaller $M_{Z^{\prime}}$ values (red points) and vice versa for the blue points. On the other hand as illustrated in the right panel, $\Omega h^{2}$ is directly proportional to the WDM mass $M_{S_{1}^{1}}$ (which is represented by the color bar). This is consistent with $\Omega h^{2}$ expression given in eq. (3.14).

In figure 8 we show the allowed points in the $\left(M_{Z^{\prime}}, g^{\prime}\right)$ and $\left(M_{S_{1}^{1}}, g^{\prime}\right)$ planes in the left and right panels, respectively, which give the relic density consistent with a relic density upper bound of the Planck measurement $\left(\Omega h^{2} \leq 0.12\right)$ [4]. All other parameter values are allowed to vary in the range mentioned in the previous paragraph. From the figure color bars (mapped to the total WDM relic density $\Omega h^{2}$ ), it is clearly seen that many points $\left(\sim 84 \%\right.$ of the scanned points) have a small DM relic density $\left(\Omega h^{2} \leq 10^{-2}\right)$. Therefore, in the next section we discuss a two component FIMP DM possibility as a well-motivated scenario to get an extra relic density contribution from the lightest heavy $\mathrm{RH}$ neutrino, $\nu_{H}^{1}$, as a GeV scale DM. 

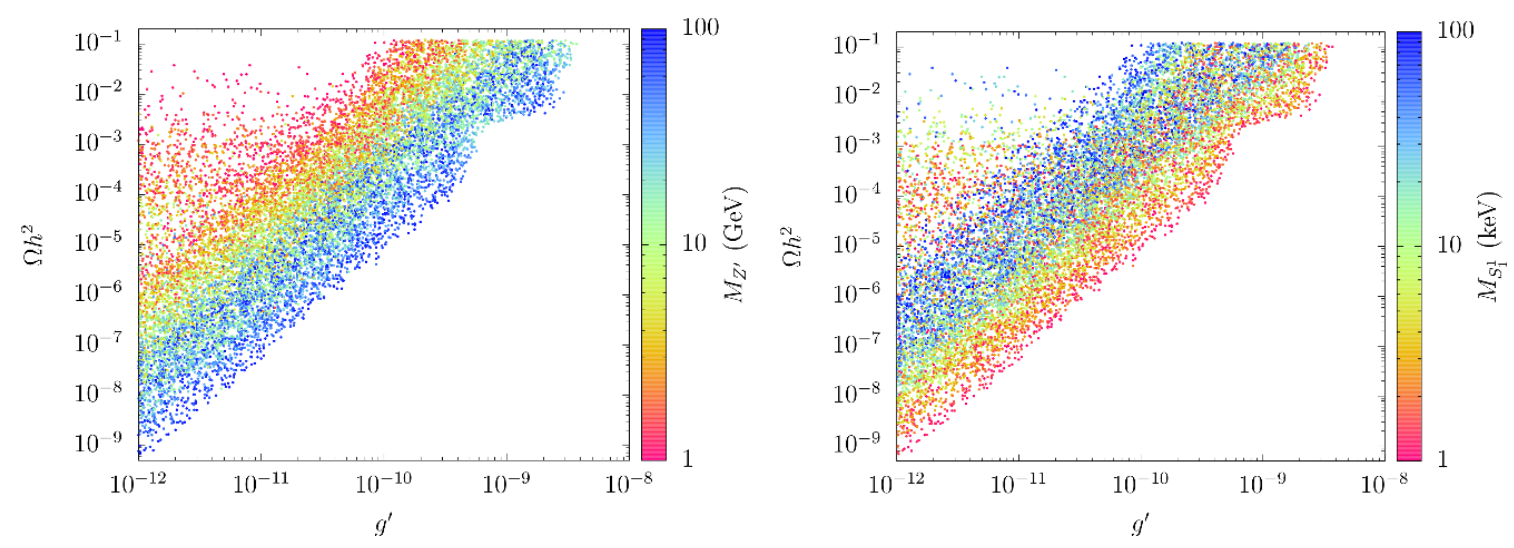

Figure 7. Allowed points in $\left(g^{\prime}, \Omega h^{2}\right)$ plane after imposing a constraint $\Omega h^{2} \leq 0.12$, as an upper bound on the WDM relic density $\Omega h^{2}$. The color bars of left and right panels correspond to the $Z^{\prime}$ mass $\left(M_{Z^{\prime}}\right)$ in $\mathrm{GeV}$ and WDM mass $\left(M_{S_{1}^{1}}\right)$ in $\mathrm{keV}$, respectively.
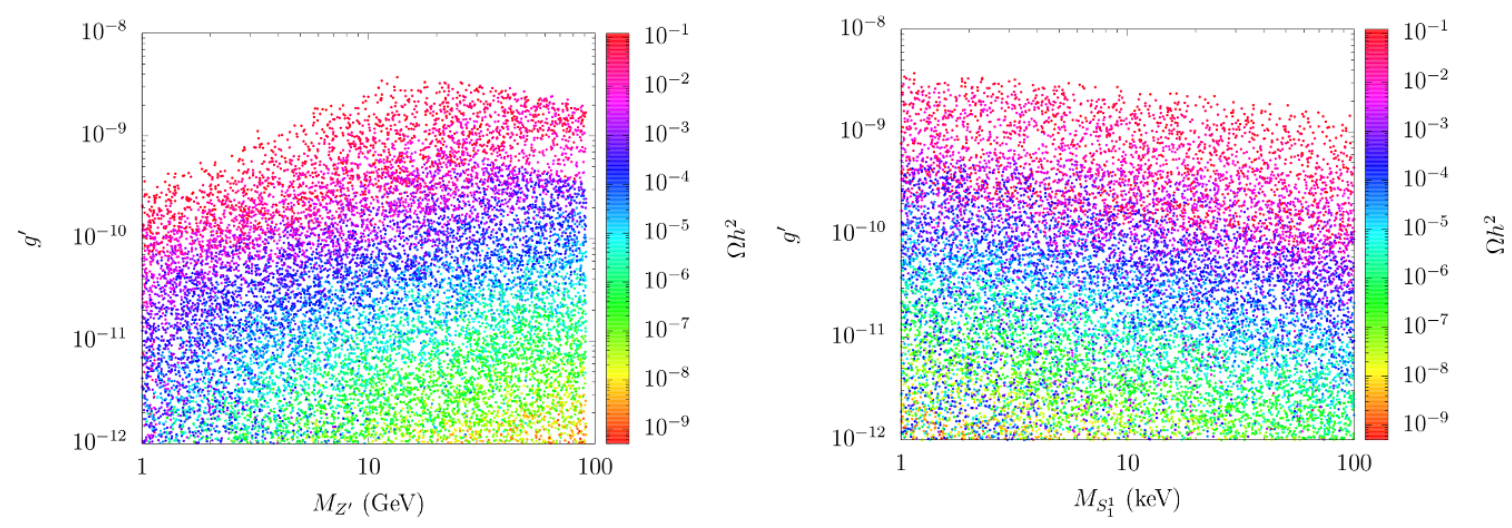

Figure 8. Allowed points in $\left(M_{Z^{\prime}}, g^{\prime}\right)$ and $\left(M_{S_{1}^{1}}, g^{\prime}\right)$ planes after imposing an upper bound on the WDM relic density $\Omega h^{2} \leq 0.12$. The color bars of left and right panels correspond to $\Omega h^{2}$.

\section{Two component FIMP dark matter}

In the previous section we have studied the WDM FIMP $S_{1}^{1}$, as a single component DM. As mentioned in section 2, the lightest heavy RH neutrino, $\nu_{H}^{1}$, can be long-lived particle by making the corresponding Yukawa couplings very small $\lesssim 3 \times 10^{-26}\left(\mathrm{GeV} / M_{N}\right)^{1 / 2}[60,61]$. Therefore, it can be an additional DM component of mass of order GeV. Note that any interaction between $S_{1}^{1}$ and $\nu_{H}^{1}$ is completely forbidden. Thus in the present section, we consider a two component DM scenario with two DM candidates: the WDM FIMP $S_{1}^{1}$ and the lightest heavy RH neutrino $\nu_{H}^{1}$. The dominant annihilation channels of $\nu_{H}^{1}$ pair to SM particles are mediated by $Z^{\prime}$ and $h_{i}(i=1,2){ }^{8}$ The coupling strength of $\nu_{H}^{1}$ pair with $Z^{\prime}$

\footnotetext{
${ }^{8}$ Due to the smallness of the corresponding Yukawa coupling of $\nu_{H}^{1}$ (as assumed to be a stable DM candidate), the contribution of the channels mediated by $Z$ and $W^{ \pm}$bosons is negligible. Also, the annihilation channels mediated by the SM-like Higgs $h_{1}$ are suppressed as compared to the $h_{2}$ ones, because the coupling $\lambda_{\nu_{H}^{1} \nu_{H}^{1} h_{1}}$ is very small since it is proportional to $\sin \alpha$ which is constrained to be very small by LHC [91].
} 
is given by $g^{\prime} / 2$, while with $h_{i}(i=1,2)$ is given as

$$
\lambda_{\nu_{H}^{1} \nu_{H}^{1} h_{i}}=\sqrt{2} g^{\prime} \frac{M_{\nu_{H}^{1}}}{M_{Z^{\prime}}} O_{i}
$$

where $O_{1}=\sin \alpha$ and $O_{2}=\cos \alpha$. Therefore, $\nu_{H}^{1}$ pair annihilation is proportional to the gauge coupling $g^{\prime}$ which is taken very small in the present model. Due to this feeble gauge coupling $g^{\prime}, \nu_{H}^{1}$ will never reach thermal equilibrium and is produced by the freeze-in mechanism. The Boltzmann equation associated with $\nu_{H}^{1}$ production is as follows

$$
\begin{aligned}
\frac{d Y_{\nu_{H}^{1}}}{d z}= & \frac{2 M_{\mathrm{Pl}} z \sqrt{g_{\star}}}{1.66 M_{\mathrm{Sc}}^{2} g_{s}}\left[\left\langle\Gamma_{Z^{\prime} \rightarrow \nu_{H}^{1} \nu_{H}^{1}}\right\rangle_{\mathrm{NTH}}\left(Y_{Z^{\prime}}-Y_{\nu_{H}^{1}}\right)+\sum_{i=1,2}\left\langle\Gamma_{h_{i}}\right\rangle\left(Y_{h_{i}}^{\mathrm{eq}}-Y_{\nu_{H}^{1}}\right)\right] \\
& +\frac{4 \pi^{2}}{45} \frac{M_{\mathrm{Pl}} M_{\mathrm{sc}} \sqrt{g_{\star}}}{1.66 z^{2}} \sum_{f}\left\langle\sigma v_{f \bar{f} \rightarrow \nu_{H}^{1} \nu_{H}^{1}}\right\rangle\left[\left(Y_{f}^{\mathrm{eq}}\right)^{2}-Y_{\nu_{H}^{1}}^{2}\right],
\end{aligned}
$$

where $\left\langle\Gamma_{Z^{\prime} \rightarrow \nu_{H}^{1} \nu_{H}^{1}}\right\rangle_{\mathrm{NTH}}$ and $\left\langle\sigma v_{f \bar{f} \rightarrow \nu_{H}^{1} \nu_{H}^{1}}\right\rangle$ are defined as in eqs. (3.10), (3.12), respectively, by replacing $S_{1}^{1}$ with $\nu_{H}^{1}$, while $Y_{h_{i}}^{\mathrm{eq}}$ is defined as in eq. (3.13) by replacing $f$ with $h_{i}$. Thermal average of the decay width of $h_{i}(i=1,2)$ is defined as [5]

$$
\left\langle\Gamma_{h_{i}}\right\rangle=\frac{K_{1}(z)}{K_{2}(z)} \Gamma_{h_{i}},
$$

where $\Gamma_{h_{i}}$ is the total decay width of $h_{i}$. After solving the Boltzmann equation of $\nu_{H}^{1}$ production, eq. (4.2), the corresponding relic density of $\nu_{H}^{1}$ can be determined by using the following relation,

$$
\Omega_{\nu_{H}^{1}} h^{2}=2.755 \times 10^{8}\left(\frac{M_{\nu_{H}^{1}}}{\mathrm{GeV}}\right) Y_{\nu_{H}^{1}}(\infty) .
$$

Finally, the total relic density of this two component DM scenario is given by

$$
\Omega^{\mathrm{tot}} h^{2}=\Omega_{\nu_{H}^{1}} h^{2}+\Omega_{S_{1}^{1}} h^{2},
$$

where $\Omega_{S_{1}^{1}} h^{2}$ is the relic density of $S_{1}^{1}$ which is defined in eq. (3.14).

It is clear that the DM production depends crucially on the DM mass and the mass of the mother particles $\left(M_{Z^{\prime}}, M_{h_{2}}\right)$. Assuming $M_{h_{2}}>2 M_{Z^{\prime}}>4 M_{S_{1}^{1}}$, we divide the $\nu_{H}^{1}$ DM spectrum into two regions according to the dominant production modes of $\nu_{H}^{1}$ DMRegion I, where $M_{Z^{\prime}}>2 M_{\nu_{H}^{1}}$ and $\nu_{H}^{1}$ production is $Z^{\prime}$ dominated, and Region II, where $M_{Z^{\prime}}<2 M_{\nu_{H}^{1}}$ and $\nu_{H}^{1}$ production is $h_{2}$ dominated.

\subsection{Region I: $M_{Z^{\prime}}>2 M_{\nu_{H}^{1}}$}

For our chosen set of BLSMIS parameters $\left(M_{Z^{\prime}}=1 \mathrm{TeV}, M_{\nu_{H}^{1}}=70 \mathrm{GeV}, M_{S_{1}^{1}}=10 \mathrm{keV}\right.$, $g^{\prime}=9.0 \times 10^{-12}, M_{h_{2}}=5 \mathrm{TeV}, \alpha=0.01 \mathrm{rad}$, and $\left.z_{0}=0.01\right)$, we show in figure 9 the variation of the total DM relic density (blue solid line) and its relative contributions for a two component DM scenario. In the figure, red solid and green dashed lines correspond 


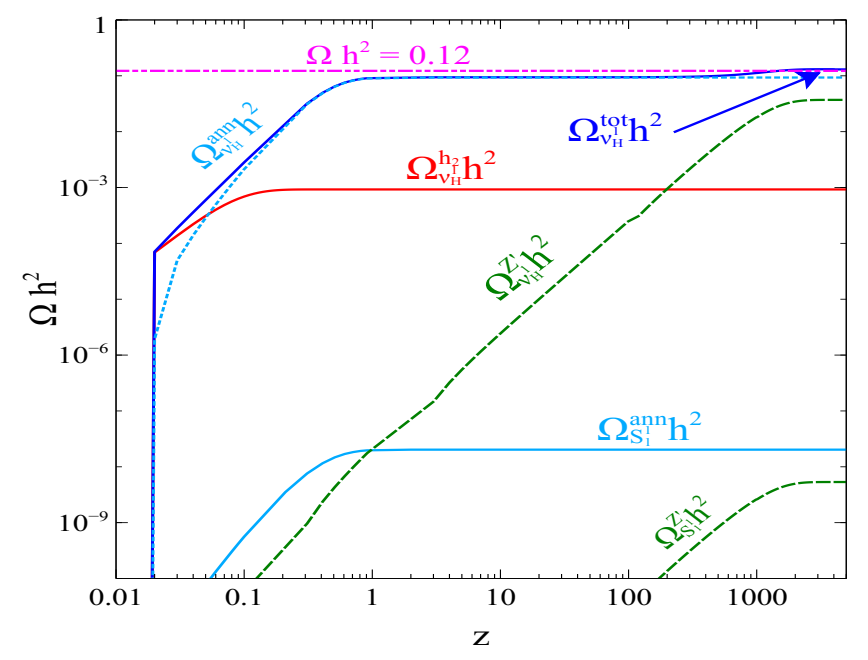

Figure 9. Variation of relic density contributions of the two DM component scenario as a function of $z$. Here, $M_{Z^{\prime}}=1 \mathrm{TeV}, M_{\nu_{H}^{1}}=70 \mathrm{GeV}, M_{S_{1}^{1}}=10 \mathrm{keV}, g^{\prime}=9.0 \times 10^{-12}, M_{h_{2}}=5 \mathrm{TeV}$, $\alpha=0.01 \mathrm{rad}$, and $z_{0}=0.01$.

to the $\nu_{H}^{1}$ relic density contributions from the decay of $h_{2}$ and $Z^{\prime}$, respectively, ${ }^{9}$ while cyan dashed line corresponds to the annihilation contribution $\left(\Omega_{\nu_{H}^{1}}^{\mathrm{ann}} h^{2}\right)$. In addition, the $S_{1}^{1}$ relic density contribution from the decay of $Z^{\prime}\left(\Omega_{S_{1}^{1}}^{Z^{\prime}} h^{2}\right)$ and annihilation $\left(\Omega_{S_{1}^{1}}^{\text {ann }} h^{2}\right)$ are presented by green dashed and cyan solid lines, respectively. Note that in region I, the relative contribution of $Z^{\prime}$ decay to $\nu_{H}^{1}$ production $\left(\Omega_{\nu_{H}^{1}}^{Z^{\prime}} h^{2}\right)$ is larger than the $h_{2}$ decay contribution $\left(\Omega_{\nu_{H}^{1}}^{h_{2}} h^{2}\right)$ because the latter is suppressed by a factor of their partial decays ratio $\left(\Gamma_{h_{2} \rightarrow \nu_{H}^{1}} \nu_{H}^{1} / \Gamma_{Z^{\prime} \rightarrow \nu_{H}^{1} \nu_{H}^{1}} \simeq 12 M_{\nu_{H}^{1}}^{2} M_{h_{2}} / M_{Z^{\prime}}^{3} \simeq \mathcal{O}(0.1)\right)$. It is also worth noting that the relic density contribution of the $\mathrm{keV} \mathrm{DM}\left(S_{1}^{1}\right)$ is negligible compared to the GeV DM $\left(\Omega_{\nu_{H}^{1}} h^{2}\right)$ even though they have the same gauge coupling strength $\left(g^{\prime}\right)$ and their mediator masses $\left(M_{h_{2}}\right.$ and $\left.M_{Z^{\prime}}\right)$ are of the same order $(\sim \mathrm{TeV})$. This is simply because the relic density of a DM candidate is directly proportional to its mass [see eqs. (3.14), (4.4)]. Therefore, the contribution of the $\mathrm{keV}$ mass $S_{1}^{1}$ to the DM total relic density is suppressed by a factor $\simeq M_{S_{1}^{1}} / M_{\nu_{H}^{1}} \simeq \mathcal{O}\left(10^{-7}\right)$ as compared to the GeV mass $\nu_{H}^{1}$.

In the left and right panels of figure 10, we show the variation of relic density contributions of the two component DM scenario for different values of $M_{Z^{\prime}}$ and $g^{\prime}$, respectively. The top panels stand for the decay contribution while the bottom ones stand for the annihilation contribution. Again from these figures, one can easily conclude that FIMP relic density contributions are inversely proportional to the mediator mass, as illustrated in left panels, and directly proportional to coupling strength as shown in right panels. We have discussed these features before in section 3 .

\footnotetext{
${ }^{9}$ Due to a smallness of the mixing angle $\alpha$, DM production of the SM-like Higgs $h_{1}$ is negligible.
} 

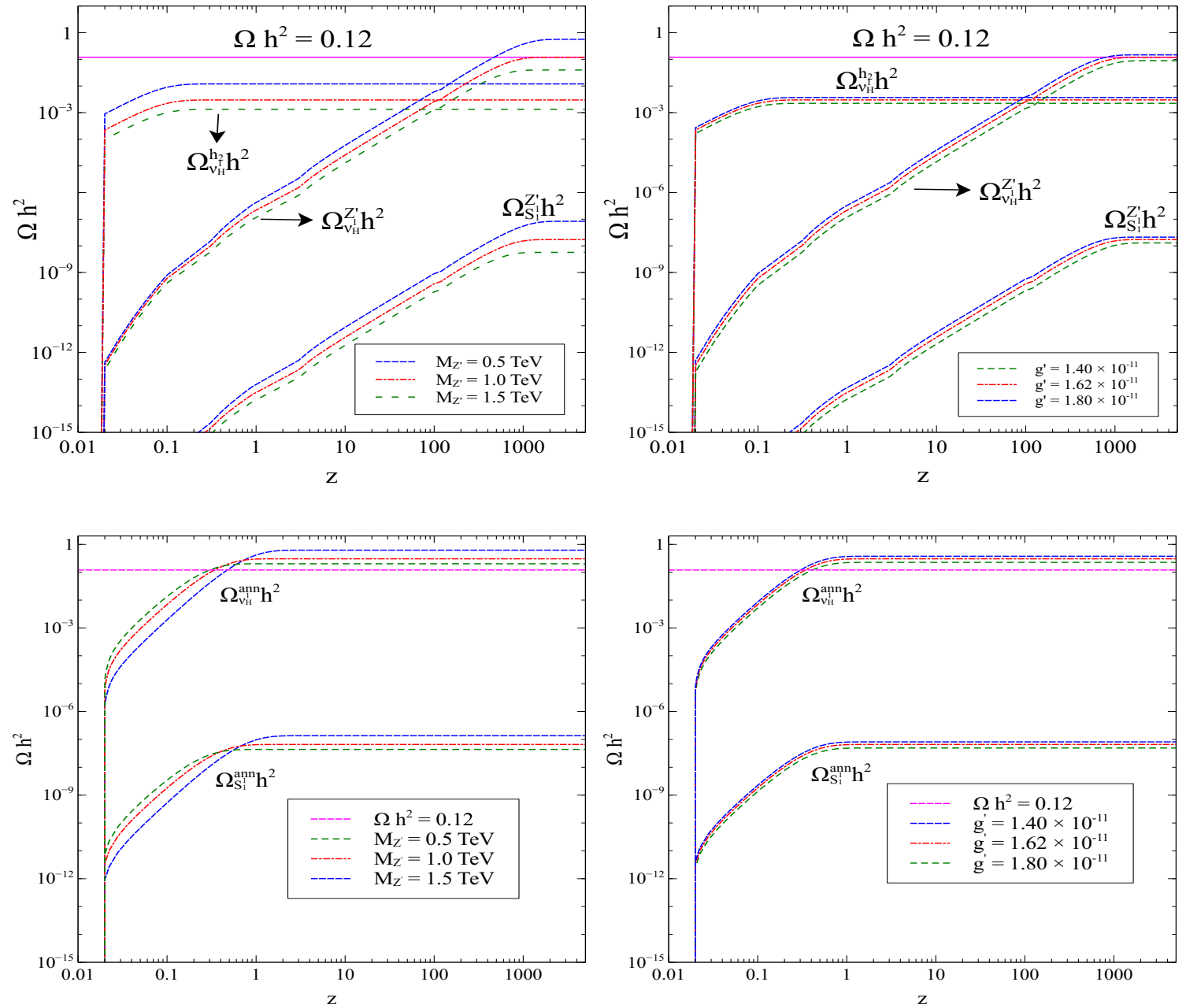

Figure 10. Variation of relative relic density contributions of a two DM component scenario as a function of $z$ for different values of $M_{Z^{\prime}}$ (left panels) and $g^{\prime}$ (right panels). Top figures correspond to decay contributions while the bottom ones correspond to annihilation contributions. Here, $M_{Z^{\prime}}=1 \mathrm{TeV}, M_{\nu_{H}^{1}}=70 \mathrm{GeV}, M_{S_{1}^{1}}=10 \mathrm{keV}, g^{\prime}=1.62 \times 10^{-11}, M_{h_{2}}=5 \mathrm{TeV}, \alpha=0.01 \mathrm{rad}$, and $z_{0}=0.01$.

\subsection{Region II: $M_{Z^{\prime}}<2 M_{\nu_{H}^{1}}$}

In discussed above, for $M_{Z^{\prime}}>2 M_{\nu_{H}^{1}}$ (region I), the total relic density is dominated by $Z^{\prime}$ mediated diagrams. Now we turn to the region II, where $M_{Z^{\prime}}<2 M_{\nu_{H}^{1}}$ and $Z^{\prime}$ decays to $\nu_{H}^{1}$ pair is kinematically forbidden, and consequently, $\nu_{H}^{1}$ production is $h_{2}$ dominated. Therefore in region II, a major portion of our two DM candidates, $\nu_{H}^{1}$ and $S_{1}^{1}$, is produced almost independently from the $h_{2}$ and $Z^{\prime}$ mediated processes, respectively. In other words, by fixing $M_{h_{2}}, g^{\prime}$ and $M_{\nu_{H}^{1}} / M_{Z^{\prime}}$ at certain values to get a significant contribution from $\Omega_{\nu_{H}^{1}} h^{2}$, one can obtain a relevant $\Omega_{S_{1}^{1}} h^{2}$ contribution independently by changing $M_{S_{1}^{1}}$ within keV range. This possibility did not exist in region I because both $\nu_{H}^{1}$ and $S_{1}^{1}$ are produced dominantly via $Z^{\prime}$ and therefore have the same number density. The only way to have comparable contribution from both in region I would be to raise the mass of $S_{1}^{1}$ to the 

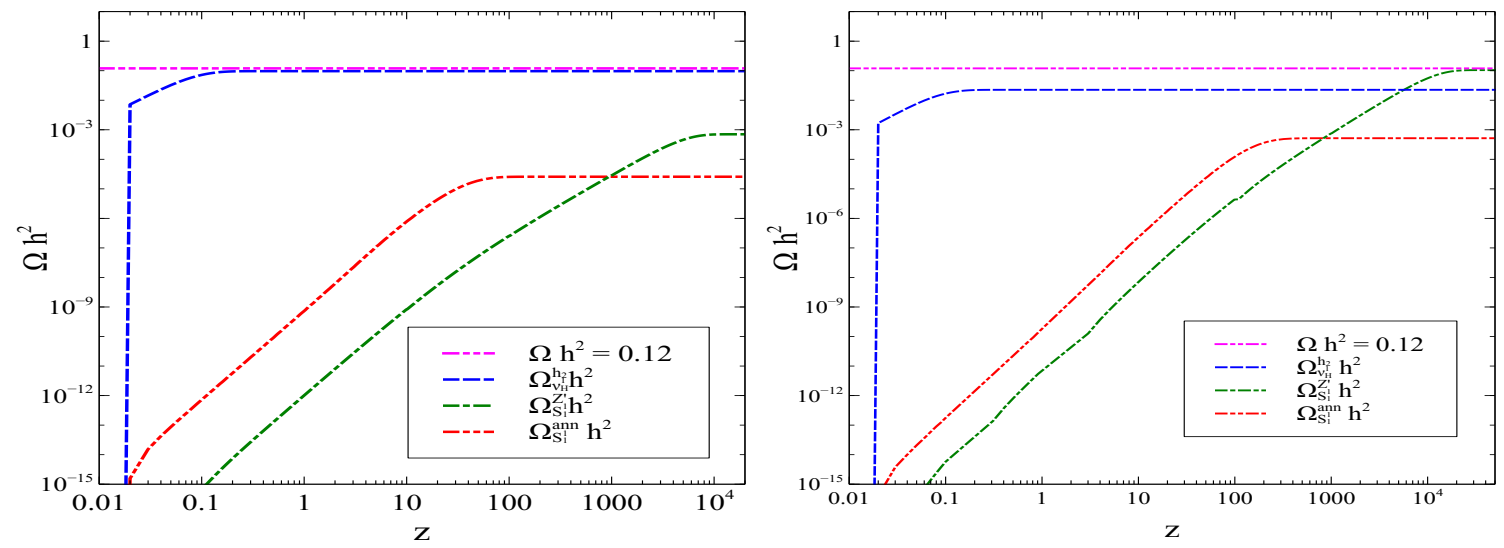

Figure 11. Variation of relative relic density contributions of $\nu_{H}^{1}$ and $S_{1}^{1}$ as a function of $z$, for two different sets of model parameters as follows. Left (right) panel: $M_{Z^{\prime}}=10 \mathrm{GeV}(2.5 \mathrm{GeV})$, $M_{\nu_{H}^{1}}=8 \mathrm{GeV}(2 \mathrm{GeV}), M_{S_{1}^{1}}=10 \mathrm{keV}(100 \mathrm{keV}), \alpha=0.01 \mathrm{rad}, g^{\prime} \simeq 2.4 \times 10^{-11}, M_{h_{2}}=5 \mathrm{TeV}$, and $z_{0}=0.01$.

$\mathrm{GeV}$ range. However, this is untenable since that will spoil the inverse seesaw mechanism scenario for generating light neutrino masses [49-52]. In region II this lacuna is remedied since here $\nu_{H}^{1}$ and $S_{1}^{1}$ are produced independently — while $\nu_{H}^{1}$ are dominantly produced from $h_{2}, S_{1}^{1}$ are produced from $Z^{\prime}$.

In figure 11, for two suitably chosen sets of BLSMIS parameters $\left(M_{Z^{\prime}}=10 \mathrm{GeV}\right.$ $(2.5 \mathrm{GeV}), M_{\nu_{H}^{1}}=8 \mathrm{GeV}(2 \mathrm{GeV}), M_{S_{1}^{1}}=10 \mathrm{keV}(100 \mathrm{keV}), \alpha=0.01 \mathrm{rad}, g^{\prime} \simeq 2.4 \times 10^{-11}$, $M_{h_{2}}=5 \mathrm{TeV}$, and $z_{0}=0.01$ ), we show variation of decay and annihilation contributions to the relic density of $S_{1}^{1}$ and $\nu_{H}^{1}$, as a function of $z$. In the figure, green dashed-dotted and red dashed-double-dotted lines correspond to the $S_{1}^{1}$ relic density contributions $\left(\Omega_{S_{1}^{1}}^{Z^{\prime}} h^{2}\right.$ and $\Omega_{S_{1}^{1}}^{\text {ann }} h^{2}$ ), respectively, while blue dashed line corresponds to $\nu_{H}^{1}$ relic density contribution from decay of $h_{2}\left(\Omega_{\nu_{H}^{1}}^{h_{2}} h^{2}\right)$. From the figure, it is clearly seen that $S_{1}^{1}$ has a relevant relic density contribution, unlike the situation in region I. Note that for a larger $S_{1}^{1}$ mass $\left(M_{S_{1}^{1}}=100 \mathrm{keV}\right)$, the $S_{1}^{1}$ contribution to the total relic density even starts to be the dominant one, as seen in the right panel of figure 11.

\section{Conclusion}

In this work we studied two beyond SM problems, viz., the non-zero neutrino masses and the existence of the DM. In studying the tiny neutrino masses, we followed the inverse seesaw mechanism within the $B-L$ extension of the SM (BLSMIS). Six SM singlet fermions were introduced for inverse seesaw mechanism to work and three more singlet fermions (with mass of order $\mathrm{keV}$ ) were added to cancel the $\mathrm{U}(1)_{B-L}$ gauge anomaly. The lightest of these additional fermionic states, $S_{1}^{1}$, can be a WDM, being odd under a $\mathbb{Z}_{2}$ discrete symmetry. We studied $S_{1}^{1}$ as a FIMP WDM and showed that it could be produced via the freeze-in mechanism from the decay of the extra neutral gauge boson $Z^{\prime}$ and the onshell annihilation processes mediated by $Z^{\prime}$. We showed that the relative contributions to 
the DM relic density from both the decay and the on-shell annihilation processes are more or less equal. We scanned over the relevant BLSMIS parameters by imposing the Planck constraint of the DM relic density and showed that a large portion of the parameter space gives a small contribution to the DM relic density. Therefore, we studied a two component FIMP DM as a possible scenario in the BLSMIS to get an extra contribution to the DM relic density. In this scenario, the lightest heavy $\mathrm{RH}$ neutrino, $\nu_{H}^{1}$, can contribute to the DM relic density as an independent DM component (with mass of order GeV). For $M_{Z^{\prime}}>2 M_{\nu_{H}^{1}}$, we showed that the production of $\nu_{H}^{1}$ as a DM candidate through the $Z^{\prime}$ mediator has the dominant contribution to the total DM relic density. On the other hand for $M_{Z^{\prime}}<2 M_{\nu_{H}^{1}}$, the $h_{2}$ mediated processes will contribute dominantly to $\nu_{H}^{1}$ production while $Z^{\prime}$ mediated processes will contribute dominantly to $S_{1}^{1}$ production. We emphasized that in this region both FIMP candidates $\left(S_{1}^{1}\right.$ and $\left.\nu_{H}^{1}\right)$ can contribute to the total DM relic density.

\section{Acknowledgments}

The authors would like to thank the Department of Atomic Energy Neutrino Project of Harish-Chandra Research Institute (HRI). Authors acknowledgment the HRI cluster computing facility (http://www.hri.res.in/cluster/). This project has received funding from the European Union's Horizon 2020 research and innovation programme InvisiblesPlus RISE under the Marie Sklodowska-Curie grant agreement No. 690575 and No. 674896. The authors would like to thank Abhass Kumar for fruitful discussions.

\section{A Analytical expression of the collision terms}

For any generic process $A(\tilde{p}) \rightarrow B\left(\tilde{p_{1}}\right) C\left(\tilde{p_{2}}\right)$ (where $\tilde{p}=\left(E_{p}, \bar{p}\right)$ ), the collision term takes the following form $[5,87]$,

$$
\begin{aligned}
\mathcal{C}\left[f_{A}(p)\right]=\frac{1}{2 E_{p}} \int & \frac{g_{B} d^{3} p_{1}}{(2 \pi)^{3} 2 E_{p_{1}}} \frac{g_{C} d^{3} p_{2}}{(2 \pi)^{3} 2 E_{p_{2}}}(2 \pi)^{4} \delta^{4}\left(\tilde{p}-\tilde{p_{1}}-\tilde{p_{2}}\right) \times \overline{|\mathcal{M}|^{2}} \\
& \times\left[f_{B} f_{C}\left(1 \pm f_{A}\right)-f_{A}\left(1 \pm f_{B}\right)\left(1 \pm f_{C}\right)\right]
\end{aligned}
$$

From eq. (3.7), we can see that the Boltzmann equation which determine the distribution function of the extra gauge boson $Z^{\prime}$ contains two collision terms one is for its production and the another one is for its decay. The expression for the two collision terms are described below.

- $\mathcal{C}^{Z^{\prime} \rightarrow \text { all }}$. Collision term for the $B-L$ gauge boson decay takes the following form after using the eq. (A.1),

$$
\mathcal{C}^{Z^{\prime} \rightarrow \text { all }}=-f_{Z^{\prime}}\left(\xi_{p}, z\right) \times \Gamma_{Z^{\prime} \rightarrow \text { all }} \times \frac{r_{Z^{\prime}}}{\sqrt{\xi_{p}^{2} \mathcal{B}(z)^{2}+r_{Z^{\prime}}^{2}}},
$$


where $r_{Z^{\prime}}=M_{Z^{\prime}} / T$ and $\Gamma_{Z^{\prime} \rightarrow \text { all }}=\Gamma_{Z^{\prime} \rightarrow f \bar{f}}+\Gamma_{Z^{\prime} \rightarrow \chi \chi}$. Expression for the each decay terms are as follows,

$$
\begin{aligned}
& \Gamma_{Z^{\prime} \rightarrow f \bar{f}}=\frac{M_{Z^{\prime}} n_{c}\left(q_{f} g^{\prime}\right)^{2}}{12 \pi}\left(1+\frac{2 M_{f}^{2}}{M_{Z^{\prime}}^{2}}\right) \sqrt{1-\frac{4 M_{f}^{2}}{M_{Z^{\prime}}^{2}}}, \\
& \Gamma_{Z^{\prime} \rightarrow \chi \chi}=\frac{M_{Z^{\prime}} g_{Z^{\prime} \chi \chi}^{2}}{24 \pi}\left(1-\frac{4 M_{\chi}^{2}}{M_{Z^{\prime}}^{2}}\right)^{3 / 2},
\end{aligned}
$$

where $f$ refers to the SM fermions and $\chi=S_{1}^{1}, \nu_{H}^{1} \cdot n_{c}$ and $q_{f}$ are the corresponding color and electric charges, respectively. $g_{Z^{\prime} \chi \chi}=g^{\prime}$ for $S_{1}^{1}$ and $g^{\prime} / 2$ for $\nu_{H}^{1}$.

- $\mathcal{C}^{h_{i} \rightarrow Z^{\prime} Z^{\prime}}$. The expression for this collision term where $h_{i}(i=1,2)$ decays to $Z^{\prime}$ pair takes the following form,

$$
\begin{aligned}
\mathcal{C}^{h_{i} \rightarrow Z^{\prime} Z^{\prime}}= & \frac{z}{48 \pi M_{\mathrm{sc}}} \frac{g_{h_{i} Z^{\prime} Z^{\prime}}^{2} \mathcal{B}^{-1}(z)}{\xi_{p} \sqrt{\xi_{p}^{2} \mathcal{B}(z)^{2}+\left(\frac{M_{Z^{\prime}} z}{M_{\mathrm{sc}}}\right)^{2}}}\left(2+\frac{\left(M_{h_{i}}^{2}-2 M_{Z^{\prime}}^{2}\right)^{2}}{4 M_{Z^{\prime}}^{4}}\right) \\
& \times\left(e^{-\sqrt{\left(\xi_{i}^{\min }\right)^{2} \mathcal{B}(z)^{2}+\left(\frac{M_{h_{i}} z}{M_{\mathrm{sc}}}\right)^{2}}}-e^{-\sqrt{\left(\xi_{i}^{\max }\right)^{2} \mathcal{B}(z)^{2}+\left(\frac{M_{h_{i}} z}{M_{\mathrm{sc}}}\right)^{2}}}\right),
\end{aligned}
$$

where

$$
\begin{aligned}
g_{h_{1} Z^{\prime} Z^{\prime}} & =\frac{2 M_{Z^{\prime}}^{2} \sin \alpha}{v^{\prime}}, \quad g_{h_{2} Z^{\prime} Z^{\prime}}=\frac{2 M_{Z^{\prime}}^{2} \cos \alpha}{v^{\prime}}, \\
\xi_{i}^{\min }\left(\xi_{p}, z\right) & =\frac{M_{\mathrm{sc}}}{2 \mathcal{B}(z) z M_{Z^{\prime}}}\left|\eta_{i}\left(\xi_{p}, z\right)-\frac{M_{h_{i}}^{2} \mathcal{B}(z)}{M_{Z^{\prime}} M_{\mathrm{sc}}} \xi_{p} z\right|, \\
\xi_{i}^{\max }\left(\xi_{p}, z\right) & =\frac{M_{\mathrm{sc}}}{2 \mathcal{B}(z) z M_{Z^{\prime}}}\left(\eta_{i}\left(\xi_{p}, z\right)+\frac{M_{h_{i}}^{2} \mathcal{B}(z)}{M_{Z^{\prime}} M_{\mathrm{sc}}} \xi_{p} z\right), \\
\eta_{i}\left(\xi_{p}, z\right) & =\frac{M_{h_{i}} z}{M_{\mathrm{sc}}} \sqrt{\frac{M_{h_{i}}^{2}}{M_{Z^{\prime}}^{2}}-4} \sqrt{\xi_{p}^{2} \mathcal{B}(z)^{2}+\left(\frac{M_{Z^{\prime}} z}{M_{\mathrm{sc}}}\right)^{2}} .
\end{aligned}
$$

- Relevant partial decay widths of the scalars $h_{i}(i=1,2)$ :

$$
\begin{aligned}
\Gamma_{h_{i} \rightarrow Z^{\prime} Z^{\prime}} & =\frac{M_{h_{i}}^{3} g^{\prime 2} O_{i}^{2}}{32 \pi M_{Z^{\prime}}^{2}} \sqrt{1-\frac{4 M_{Z^{\prime}}^{2}}{M_{h_{i}}^{2}}}\left(1-\frac{4 M_{Z^{\prime}}^{2}}{M_{h_{i}}^{2}}+\frac{12 M_{Z^{\prime}}^{4}}{M_{h_{i}}^{4}}\right), \\
\Gamma_{h_{i} \rightarrow \nu_{H}^{1} \nu_{H}^{1}} & =\frac{M_{h_{i}} \lambda_{\nu_{H}^{1} \nu_{H}^{1} h_{i}}^{2}}{16 \pi}\left(1-\frac{4 M_{\nu_{H}^{1}}^{2}}{M_{h_{i}}^{2}}\right)^{3 / 2},
\end{aligned}
$$

where $O_{1}=\sin \alpha, O_{2}=\cos \alpha$, and the coupling $\lambda_{\nu_{H}^{1}} \nu_{H}^{1} h_{i}$ is defined in eq. (4.1). 
Open Access. This article is distributed under the terms of the Creative Commons Attribution License (CC-BY 4.0), which permits any use, distribution and reproduction in any medium, provided the original author(s) and source are credited.

\section{References}

[1] F. Zwicky, Die Rotverschiebung von extragalaktischen Nebeln, Helv. Phys. Acta 6 (1933) 110 [INSPIRE].

[2] V.C. Rubin and W.K. Ford Jr., Rotation of the Andromeda Nebula from a Spectroscopic Survey of Emission Regions, Astrophys. J. 159 (1970) 379 [INSPIRE].

[3] D. Clowe et al., A direct empirical proof of the existence of dark matter, Astrophys. J. 648 (2006) L109 [astro-ph/0608407] [INSPIRE].

[4] PlancK collaboration, Planck 2015 results. XIII. Cosmological parameters, Astron. Astrophys. 594 (2016) A13 [arXiv:1502.01589] [INSPIRE].

[5] P. Gondolo and G. Gelmini, Cosmic abundances of stable particles: Improved analysis, Nucl. Phys. B 360 (1991) 145 [INSPIRE].

[6] M. Srednicki, R. Watkins and K.A. Olive, Calculations of Relic Densities in the Early Universe, Nucl. Phys. B 310 (1988) 693 [InSPIRE].

[7] XENON collaboration, Dark Matter Search Results from a One Ton-Year Exposure of XENON1T, Phys. Rev. Lett. 121 (2018) 111302 [arXiv:1805.12562] [INSPIRE].

[8] PandaX-II collaboration, Dark Matter Results from First 98.7 Days of Data from the PandaX-II Experiment, Phys. Rev. Lett. 117 (2016) 121303 [arXiv:1607.07400] [INSPIRE].

[9] LUX collaboration, Results from a search for dark matter in the complete LUX exposure, Phys. Rev. Lett. 118 (2017) 021303 [arXiv:1608.07648] [INSPIRE].

[10] PICO collaboration, Dark Matter Search Results from the PICO-60 $C_{3} F_{8}$ Bubble Chamber, Phys. Rev. Lett. 118 (2017) 251301 [arXiv:1702.07666] [INSPIRE].

[11] XENON collaboration, Constraining the spin-dependent WIMP-nucleon cross sections with XENON1T, Phys. Rev. Lett. 122 (2019) 141301 [arXiv:1902.03234] [INSPIRE].

[12] MAGIC and Fermi-LAT collaborations, Limits to Dark Matter Annihilation Cross-Section from a Combined Analysis of MAGIC and Fermi-LAT Observations of Dwarf Satellite Galaxies, JCAP 02 (2016) 039 [arXiv:1601.06590] [INSPIRE].

[13] H.E.S.S. collaboration, Search for dark matter annihilations towards the inner Galactic halo from 10 years of observations with H.E.S.S, Phys. Rev. Lett. 117 (2016) 111301 [arXiv: 1607.08142] [INSPIRE].

[14] G. Giesen et al., AMS-02 antiprotons, at last! Secondary astrophysical component and immediate implications for Dark Matter, JCAP 09 (2015) 023 [arXiv:1504.04276] [INSPIRE].

[15] A. Drukier and L. Stodolsky, Principles and Applications of a Neutral Current Detector for Neutrino Physics and Astronomy, Phys. Rev. D 30 (1984) 2295 [InSPIRE].

[16] P. Cushman et al., Working Group Report: WIMP Dark Matter Direct Detection, in Proceedings, 2013 Community Summer Study on the Future of U.S. Particle Physics: Snowmass on the Mississippi (CSS2013), Minneapolis, MN, U.S.A., July 29-August 6, 2013 (2013) [arXiv: 1310.8327] [INSPIRE]. 
[17] L.J. Hall, K. Jedamzik, J. March-Russell and S.M. West, Freeze-In Production of FIMP Dark Matter, JHEP 03 (2010) 080 [arXiv:0911.1120] [INSPIRE].

[18] J. König, A. Merle and M. Totzauer, keV Sterile Neutrino Dark Matter from Singlet Scalar Decays: The Most General Case, JCAP 11 (2016) 038 [arXiv:1609.01289] [INSPIRE].

[19] A. Biswas and A. Gupta, Freeze-in Production of Sterile Neutrino Dark Matter in $\mathrm{U}(1)_{B-L}$ Model, JCAP 09 (2016) 044 [arXiv: 1607.01469] [INSPIRE].

[20] A. Biswas and A. Gupta, Calculation of Momentum Distribution Function of a Non-thermal Fermionic Dark Matter, JCAP 03 (2017) 033 [arXiv:1612.02793] [INSPIRE].

[21] A. Biswas, S. Choubey and S. Khan, FIMP and Muon $(g-2)$ in a $\mathrm{U}(1)_{L_{\mu}-L_{\tau}}$ Model, JHEP 02 (2017) 123 [arXiv: 1612.03067] [INSPIRE].

[22] A. Biswas, S. Choubey and S. Khan, Neutrino mass, leptogenesis and FIMP dark matter in $a \mathrm{U}(1)_{B-L}$ model, Eur. Phys. J. C 77 (2017) 875 [arXiv:1704.00819] [INSPIRE].

[23] A. Biswas, S. Choubey, L. Covi and S. Khan, Explaining the $3.5 \mathrm{keV} X$-ray Line in a $L_{\mu}-L_{\tau}$ Extension of the Inert Doublet Model, JCAP 02 (2018) 002 [arXiv:1711.00553] [INSPIRE].

[24] K. Kaneta, Z. Kang and H.-S. Lee, Right-handed neutrino dark matter under the B $-L$ gauge interaction, JHEP 02 (2017) 031 [arXiv: 1606.09317] [INSPIRE].

[25] A. Caputo, P. Hernández and N. Rius, Low-scale leptogenesis and dark matter, arXiv: 1807.03309 [INSPIRE].

[26] C.L. Cowan, F. Reines, F.B. Harrison, H.W. Kruse and A.D. McGuire, Detection of the free neutrino: A Confirmation, Science 124 (1956) 103 [INSPIRE].

[27] Super-KamiokAnde collaboration, Evidence for oscillation of atmospheric neutrinos, Phys. Rev. Lett. 81 (1998) 1562 [hep-ex/9807003] [INSPIRE].

[28] SNO collaboration, Direct evidence for neutrino flavor transformation from neutral current interactions in the Sudbury Neutrino Observatory, Phys. Rev. Lett. 89 (2002) 011301 [nucl-ex/0204008] [INSPIRE].

[29] KAMLAND collaboration, First results from KamLAND: Evidence for reactor anti-neutrino disappearance, Phys. Rev. Lett. 90 (2003) 021802 [hep-ex/0212021] [INSPIRE].

[30] DAYA BAY collaboration, Measurement of the Reactor Antineutrino Flux and Spectrum at Daya Bay, Phys. Rev. Lett. 116 (2016) 061801 [Erratum ibid. 118 (2017) 099902] [arXiv: 1508.04233] [INSPIRE].

[31] RENO collaboration, Observation of Energy and Baseline Dependent Reactor Antineutrino Disappearance in the RENO Experiment, Phys. Rev. Lett. 116 (2016) 211801 [arXiv: 1511.05849] [INSPIRE].

[32] Double CHOOZ collaboration, Improved measurements of the neutrino mixing angle $\theta_{13}$ with the Double CHOOZ detector, JHEP 10 (2014) 086 [Erratum ibid. 02 (2015) 074] [arXiv: 1406.7763] [INSPIRE].

[33] T2K collaboration, Measurements of neutrino oscillation in appearance and disappearance channels by the T2K experiment with $6.6 \times 10^{20}$ protons on target, Phys. Rev. D 91 (2015) 072010 [arXiv: 1502.01550] [INSPIRE].

[34] T2K collaboration, Anti-neutrino oscillations with T2K, arXiv:1508.06153 [INSPIRE].

[35] NOvA collaboration, First measurement of electron neutrino appearance in NOvA, Phys. Rev. Lett. 116 (2016) 151806 [arXiv: 1601.05022] [INSPIRE]. 
[36] NOvA collaboration, First measurement of muon-neutrino disappearance in NOvA, Phys. Rev. D 93 (2016) 051104 [arXiv:1601.05037] [INSPIRE].

[37] S. Khalil, Low scale B-L extension of the Standard Model at the LHC, J. Phys. G 35 (2008) 055001 [hep-ph/0611205] [INSPIRE].

[38] W. Emam and S. Khalil, Higgs and $Z^{\prime}$ phenomenology in $B-L$ extension of the standard model at LHC, Eur. Phys. J. C 52 (2007) 625 [arXiv:0704.1395] [InSPIRE].

[39] S. Blanchet, Z. Chacko, S.S. Granor and R.N. Mohapatra, Probing Resonant Leptogenesis at the LHC, Phys. Rev. D 82 (2010) 076008 [arXiv:0904.2174] [InSPIRE].

[40] L. Basso, A. Belyaev, S. Moretti and C.H. Shepherd-Themistocleous, Phenomenology of the minimal B $-L$ extension of the Standard model: $Z^{\prime}$ and neutrinos, Phys. Rev. D 80 (2009) 055030 [arXiv:0812.4313] [INSPIRE].

[41] K. Huitu, S. Khalil, H. Okada and S.K. Rai, Signatures for right-handed neutrinos at the Large Hadron Collider, Phys. Rev. Lett. 101 (2008) 181802 [arXiv:0803.2799] [INSPIRE].

[42] R.N. Mohapatra and R.E. Marshak, Local B - L Symmetry of Electroweak Interactions, Majorana Neutrinos and Neutron Oscillations, Phys. Rev. Lett. 44 (1980) 1316 [Erratum ibid. 44 (1980) 1643] [INSPIRE].

[43] R.E. Marshak and R.N. Mohapatra, Quark-Lepton Symmetry and B - L as the U(1) Generator of the Electroweak Symmetry Group, Phys. Lett. 91B (1980) 222 [INSPIRE].

[44] C. Wetterich, Neutrino Masses and the Scale of B-L Violation, Nucl. Phys. B 187 (1981) 343 [INSPIRE].

[45] A. Masiero, J.F. Nieves and T. Yanagida, B $-L$ Violating Proton Decay and Late Cosmological Baryon Production, Phys. Lett. 116B (1982) 11 [INSPIRE].

[46] R.N. Mohapatra and G. Senjanović, Spontaneous Breaking of Global B - L Symmetry and Matter-Antimatter Oscillations in Grand Unified Theories, Phys. Rev. D 27 (1983) 254 [INSPIRE].

[47] W. Buchmüller, C. Greub and P. Minkowski, Neutrino masses, neutral vector bosons and the scale of B-L breaking, Phys. Lett. B 267 (1991) 395 [INSPIRE].

[48] M. Abbas and S. Khalil, Neutrino masses, mixing and leptogenesis in TeV scale B - L extension of the standard model, JHEP 04 (2008) 056 [arXiv:0707.0841] [INSPIRE].

[49] S. Khalil, TeV-scale gauged B - L symmetry with inverse seesaw mechanism, Phys. Rev. D 82 (2010) 077702 [arXiv: 1004.0013] [INSPIRE].

[50] R.N. Mohapatra, Mechanism for Understanding Small Neutrino Mass in Superstring Theories, Phys. Rev. Lett. 56 (1986) 561 [InSPIRE].

[51] R.N. Mohapatra and J.W.F. Valle, Neutrino Mass and Baryon Number Nonconservation in Superstring Models, Phys. Rev. D 34 (1986) 1642 [INSPIRE].

[52] M.C. Gonzalez-Garcia and J.W.F. Valle, Fast Decaying Neutrinos and Observable Flavor Violation in a New Class of Majoron Models, Phys. Lett. B 216 (1989) 360 [INSPIRE].

[53] G. 't Hooft, Naturalness, chiral symmetry, and spontaneous chiral symmetry breaking, NATO Sci. Ser. B 59 (1980) 135 [INSPIRE].

[54] H. Pagels and J.R. Primack, Supersymmetry, Cosmology and New TeV Physics, Phys. Rev. Lett. 48 (1982) 223 [inSPIRE]. 
[55] P.J.E. Peebles, Primeval adiabatic perturbations: effect of massive neutrinos, Astrophys. J. 258 (1982) 415 [INSPIRE].

[56] J.R. Bond, A.S. Szalay and M.S. Turner, Formation of Galaxies in a Gravitino Dominated Universe, Phys. Rev. Lett. 48 (1982) 1636 [InSPIRE].

[57] K.A. Olive and M.S. Turner, Cosmological Bounds on the Masses of Stable, Right-handed Neutrinos, Phys. Rev. D 25 (1982) 213 [inSPIRE].

[58] A. El-Zant, S. Khalil and A. Sil, Warm dark matter in a $B-L$ inverse seesaw scenario, Phys. Rev. D 91 (2015) 035030 [arXiv:1308.0836] [inSPIRE].

[59] A. Boyarsky, O. Ruchayskiy and M. Shaposhnikov, The Role of sterile neutrinos in cosmology and astrophysics, Ann. Rev. Nucl. Part. Sci. 59 (2009) 191 [arXiv:0901.0011] [INSPIRE].

[60] M. Re Fiorentin, V. Niro and N. Fornengo, A consistent model for leptogenesis, dark matter and the IceCube signal, JHEP 11 (2016) 022 [arXiv:1606.04445] [INSPIRE].

[61] P. Di Bari, P.O. Ludl and S. Palomares-Ruiz, Unifying leptogenesis, dark matter and high-energy neutrinos with right-handed neutrino mixing via Higgs portal, JCAP 11 (2016) 044 [arXiv: 1606. 06238] [INSPIRE].

[62] B. Holdom, Two U(1)'s and Epsilon Charge Shifts, Phys. Lett. 166B (1986) 196 [INSPIRE].

[63] P.H. Chankowski, S. Pokorski and J. Wagner, $Z^{\prime}$ and the Appelquist-Carrazzone decoupling, Eur. Phys. J. C 47 (2006) 187 [hep-ph/0601097] [InSPIRE].

[64] M.E. Krauss, B. O'Leary, W. Porod and F. Staub, Implications of gauge kinetic mixing on $Z^{\prime}$ and slepton production at the LHC, Phys. Rev. D 86 (2012) 055017 [arXiv:1206.3513] [INSPIRE].

[65] DELPHI collaboration, A Study of radiative muon pair events at $Z^{0}$ energies and limits on an additional $Z^{\prime}$ gauge boson, Z. Phys. C 65 (1995) 603 [inSPIRE].

[66] ALEPH, DELPHI, L3 and OPAL collaborations, LEP Electroweak Working Group, $A$ Combination of preliminary electroweak measurements and constraints on the standard model, hep-ex/0612034 [INSPIRE].

[67] J. Erler, P. Langacker, S. Munir and E. Rojas, Improved Constraints on $Z^{\prime}$ Bosons from Electroweak Precision Data, JHEP 08 (2009) 017 [arXiv: 0906. 2435] [INSPIRE].

[68] G. Cacciapaglia, C. Csáki, G. Marandella and A. Strumia, The Minimal Set of Electroweak Precision Parameters, Phys. Rev. D 74 (2006) 033011 [hep-ph/0604111] [InSPIRE].

[69] M. Carena, A. Daleo, B.A. Dobrescu and T.M.P. Tait, $Z^{\prime}$ gauge bosons at the Tevatron, Phys. Rev. D 70 (2004) 093009 [hep-ph/0408098] [InSPIRE].

[70] W. Abdallah, A. Awad, S. Khalil and H. Okada, Muon Anomalous Magnetic Moment and $\mu \rightarrow$ er in B - L Model with Inverse Seesaw, Eur. Phys. J. C 72 (2012) 2108 [arXiv: 1105.1047] [INSPIRE].

[71] E. Ma, Radiative inverse seesaw mechanism for nonzero neutrino mass, Phys. Rev. D 80 (2009) 013013 [arXiv:0904.4450] [INSPIRE].

[72] A.A. Abdelalim, A. Hammad and S. Khalil, B - L heavy neutrinos and neutral gauge boson $Z^{\prime}$ at the LHC, Phys. Rev. D 90 (2014) 115015 [arXiv:1405.7550] [INSPIRE].

[73] P. Bandyopadhyay, E.J. Chun, H. Okada and J.-C. Park, Higgs Signatures in Inverse Seesaw Model at the LHC, JHEP 01 (2013) 079 [arXiv: 1209.4803] [INSPIRE]. 
[74] W. Abdallah, D. Delepine and S. Khalil, TeV Scale Leptogenesis in B - L Model with Alternative Cosmologies, Phys. Lett. B 725 (2013) 361 [arXiv:1205.1503] [InSPIRE].

[75] D. Hooper, F.S. Queiroz and N.Y. Gnedin, Non-Thermal Dark Matter Mimicking An Additional Neutrino Species In The Early Universe, Phys. Rev. D 85 (2012) 063513 [arXiv:1111.6599] [INSPIRE].

[76] ATLAS collaboration, Observation of a new particle in the search for the Standard Model Higgs boson with the ATLAS detector at the LHC, Phys. Lett. B 716 (2012) 1 [arXiv: 1207.7214] [INSPIRE].

[77] CMS collaboration, Observation of a new boson at a mass of $125 \mathrm{GeV}$ with the CMS experiment at the LHC, Phys. Lett. B 716 (2012) 30 [arXiv:1207.7235] [INSPIRE].

[78] L. Basso, S. Moretti and G.M. Pruna, A Renormalisation Group Equation Study of the Scalar Sector of the Minimal B - L Extension of the Standard Model, Phys. Rev. D 82 (2010) 055018 [arXiv: 1004.3039] [INSPIRE].

[79] F. Staub, SARAH 4: A tool for (not only SUSY) model builders, Comput. Phys. Commun. 185 (2014) 1773 [arXiv:1309.7223] [INSPIRE].

[80] F. Staub, From Superpotential to Model Files for FeynArts and CalcHep/CompHEP, Comput. Phys. Commun. 181 (2010) 1077 [arXiv:0909.2863] [INSPIRE].

[81] F. Staub, SARAH, arXiv:0806.0538 [INSPIRE].

[82] W. Porod and F. Staub, SPheno 3.1: Extensions including flavour, CP-phases and models beyond the MSSM, Comput. Phys. Commun. 183 (2012) 2458 [arXiv:1104.1573] [INSPIRE].

[83] M. Drewes et al., A White Paper on keV Sterile Neutrino Dark Matter, JCAP 01 (2017) 025 [arXiv: 1602.04816] [INSPIRE].

[84] N. Bernal, M. Heikinheimo, T. Tenkanen, K. Tuominen and V. Vaskonen, The Dawn of FIMP Dark Matter: A Review of Models and Constraints, Int. J. Mod. Phys. A 32 (2017) 1730023 [arXiv: 1706.07442] [INSPIRE].

[85] A. Abada, G. Arcadi and M. Lucente, Dark Matter in the minimal Inverse Seesaw mechanism, JCAP 10 (2014) 001 [arXiv:1406.6556] [INSPIRE].

[86] G. Arcadi and L. Covi, Minimal Decaying Dark Matter and the LHC, JCAP 08 (2013) 005 [arXiv:1305.6587] [INSPIRE].

[87] E.W. Kolb and M.S. Turner, The Early Universe, Front. Phys. 69 (1990) 1 [InSPIRE].

[88] G. Bélanger, F. Boudjema, A. Goudelis, A. Pukhov and B. Zaldivar, MicrOMEGAs5.0: Freeze-in, Comput. Phys. Commun. 231 (2018) 173 [arXiv: 1801.03509] [INSPIRE].

[89] J. Edsjo and P. Gondolo, Neutralino relic density including coannihilations, Phys. Rev. D 56 (1997) 1879 [hep-ph/9704361] [INSPIRE].

[90] S. Iso, N. Okada and Y. Orikasa, Resonant Leptogenesis in the Minimal B - L Extended Standard Model at TeV, Phys. Rev. D 83 (2011) 093011 [arXiv:1011.4769] [INSPIRE].

[91] ATLAS and CMS collaborations, Measurements of the Higgs boson production and decay rates and constraints on its couplings from a combined ATLAS and CMS analysis of the LHC pp collision data at $\sqrt{s}=7$ and $8 \mathrm{TeV}$, JHEP 08 (2016) 045 [arXiv:1606.02266] [INSPIRE]. 